Preprints of the

Max Planck Institute for

Research on Collective Goods

Bonn 2012/20

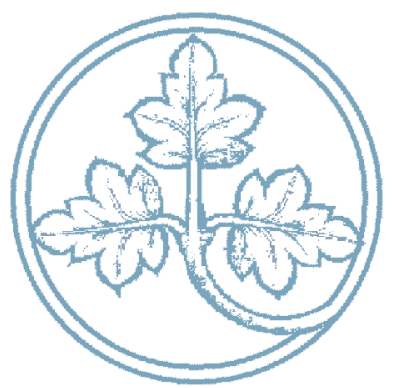

Divided Majority and

Information Aggregation:

Theory and Experiment

Laurent Bouton

Micael Castanheira

Aniol Llorente-Saguer

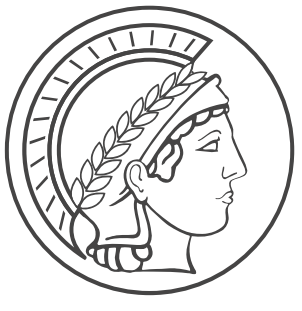




\title{
Divided Majority and Information Aggregation: Theory and Experiment
}

\author{
Laurent Bouton / Micael Castanheira / Aniol Llorente-Saguer
}

November 2012 


\title{
Divided Majority and Information
}

\section{Aggregation: Theory and Experiment*}

\author{
Laurent Bouton Micael Castanheira \\ Aniol Llorente-Saguer \\ Boston University Univestité Libre \\ Max Planck Institute for \\ de Bruxelles Research on Collective Goods
}

November 19, 2012

\begin{abstract}
This paper both theoretically and experimentally studies the properties of plurality and approval voting when the majority is divided as a result of information imperfections. The minority backs a third alternative, which the majority views as strictly inferior. The majority thus faces two problems: aggregating information and coordinating to defeat the minority candidate. Two types of equilibria coexist under plurality: either voters aggregate information, but this requires splitting their votes, or they coordinate but cannot aggregate information. With approval voting, expected welfare is strictly higher, because some voters multiple vote to achieve both goals at once. In the laboratory, we observe both types of equilibrium under plurality. Which one is selected depends on the size of the minority. Approval voting vastly outperforms plurality. Finally, subject behavior suggests the need to study asymmetric equilibria.
\end{abstract}

JEL Classification: C72, C92, D70, P16

Keywords: Multicandidate Elections, Plurality, Approval Voting, Experiments

${ }^{*}$ We thank participants to the ESA 2012 meetings in Tucson, the Political Economy Workshop at the Erasmus University Rotterdam, the Workshop on Social Protests and Political Influence and seminars at Boston University, Columbia, CREED, IMT Lucca, London School of Economics, Massachussets Institute of Technology, New York University, Oxford, Pittsburgh, Queen Mary, Royal Holloway, Tilburg and Warwick. We particularly thank Alessandra Casella, Eric Van Damme, Christoph Engel, Olga Gorelkina, Kristoffel Grechenig, Alessandro Lizzeri, Roger Myerson, Tom Palfrey and Jean-Benoit Pilet. We would also like to thank Erika Gross and Nicolas Meier for excellent assistance at running the experiments. We gratefully acknowledge financial support from the Max Planck Society. Micael Castanheira is a senior research fellow of the Fonds National de la Recherche Scientifique and is grateful for their support. 


\section{Introduction}

Elections are typically expected to achieve better-informed decisions than what a single individual could achieve alone (Condorcet 1785, Austen-Smith and Banks 1996, Feddersen and Pesendorfer 1997, 1998, etc). The rationale is that each single individual only has a fraction of the overall information held by the electorate. If each voter can convey her privately-held information through her ballot, voting results will reveal the aggregate information held by the electorate. However, this is a big "if": in plurality, for instance, rational voters are typically expected to coordinate their ballots on only two alternatives, independently of the number of competing alternatives (Duverger's Law). Therefore, unless the number of candidates is exactly two, information aggregation is jeopardized.

This impossibility resonates with centuries of scholarly research on how to design an electoral system that would be better able to aggregate heterogeneous preferences and information in an efficacious way (see e.g. Condorcet 1785, Borda 1781, Arrow 1951, Cox 1997, Myerson and Weber 1993, and Myerson 2002). Frustration with plurality is also apparent in civil society: a large number of activists lobby in favor of reforming the electoral system ${ }^{1}$ and many reform proposals have been officially introduced. ${ }^{2}$ One of the most supported alternatives to plurality is approval voting (AV). ${ }^{3}$ Yet a major hurdle stands in the way of any reform of the electoral system: the substantial lack of knowledge surrounding the capacity of AV (or other systems) to outperform plurality. ${ }^{4}$ We need a better understanding of the properties of each potential electoral system to identify and implement meaningful reforms.

With this purpose in mind, this paper studies the properties of plurality and AV when voters are strategic but imperfectly informed. Our analysis features two main novelties: first, we study these systems both theoretically and experimentally. We focus on the case

\footnotetext{
${ }^{1}$ See e.g. the Electoral Reform Society (www.electoral-reform.org.uk) and the Fair Vote Reforms initiative (www.fairvote.org).

${ }^{2}$ Two examples are North Dakota in 1987, where a bill to enact approval voting in some statewide elections passed the Senate but not the House and, more recently, the U.K., which held a national referendum in 2011 on whether to replace plurality voting with alternative voting.

${ }^{3}$ Under approval voting, voters can "approve of" as many candidates as they want, each approval counts as one vote and the candidate that obtains the largest number of votes wins (Weber 1977, 1995, Brams and Fishburn 1978, 1983, Laslier 2009).

${ }^{4}$ For instance, the 2011 referendum in the U.K. arguably rejected the implementation of the proposed substitute, alternative voting, largely because of the uncertainty surrounding its capacity to outperform plurality.
} 
in which a majority needs to both aggregate information and coordinate ballots to defeat a minority alternative, the Condorcet loser. Second, instead of focusing on the limiting properties of these systems when the electorate is arbitrarily large, we study them for any electorate size. This means that our conclusions are equally valid from committees to general elections.

A first theoretical finding is that, in plurality, the need to aggregate information produces an equilibrium in which voters vote informatively (that is, their ballot conveys information about their beliefs, e.g. because they vote sincerely), despite the need to coordinate against the minority. This equilibrium is not "knife edge", and may rationalize the oft-observed pattern that strictly more than two candidates receive positive but different vote shares, despite the prediction of Duverger's Law (Duverger 1963, Palfrey 1989, Myerson and Weber 1993, Cox 1997, and Fey 1997). When the minority is small, this equilibrium supports information aggregation, in the sense that the alternative with the largest expected vote share is the full information Condorcet winner. In contrast, when the minority is large, the alternative with the largest vote share is the Condorcet loser, in which case this equilibrium is highly inefficient. This informative equilibrium may thus exist despite the fact that majority voters would benefit from collectively deviating towards a two-alternative equilibrium. That is, Duverger's Law may or may not be observed in equilibrium, depending on how majority voters coordinate their ballots.

In our divided majority setup, we also find that AV can always produce strictly higher welfare than plurality: for any equilibrium in plurality, allowing voters to approve of more than one alternative produces a two-pronged benefit: first, it reduces the threat posed by the minority alternative. Second, voters in the majority can better aggregate information. While characterizing the exact equilibrium in approval voting is not possible for any electorate size,$^{5}$ we are able to formulate two substantiated conjectures: (i) the equilibrium is unique, and (ii) the equilibrium strategy is such that majority voters always approve of the candidate they deem best and sometimes also approve of the other majority candidate. These conjectures find support in one formal result and many numerical simulations. First, we prove that voters must adopt this voting pattern in any interior equilibrium (in

\footnotetext{
${ }^{5}$ In contrast, in a related setup, Bouton and Castanheira (2012) fully characterize the equilibrium for arbitrarily large electorate sizes. The equilibrium is then unique and it implies that the full information Condorcet winner always has the largest expected vote share.
} 
which voters play nondegenerate mixed strategies). Second, for all the parametric values we checked, the equilibrium was indeed unique.

These theoretical results pose an interesting trade-off between these two electoral mechanisms. On the one hand, AV is inherently more complex than plurality since it extends the set of actions that each voter can take. ${ }^{6}$ A risk exists then that actual voters make more mistakes at the time of voting, which could wash out the favorable theoretical properties of AV. On the other hand, our theoretical findings are that AV reduces the number of equilibria and therefore simplifies strategic interactions amongst voters when they have imperfect information. In other words, AV should facilitate the voters' two-pronged goal of aggregating information and coordinating ballots to avoid a victory of the Condorcet loser.

To assess the validity of these theoretical findings, we have run a series of laboratory experiments. Through these, we can evaluate the effective performance of each system in a controlled environment. Equally important, these experiments allow us to bring new light to the debates on voter rationality: determining whether voters behave strategically and respond to incentives is a central issue in the quest for better political institutions. Our setup, which combines the need to (i) aggregate information, and (ii) coordinate ballots, is an ideal testing ground for such questions. First, each of these two problems produce different -often opposite- voting incentives. The literature has extensively studied each of these problems, but typically in isolation (see subsection 1.1). Combining the two allows us to test whether and in which proportion voters react to a change in incentives when we modify the relative value of coordinating ballots versus aggregating information. Second, studying multicandidate rather than two-candidate elections widens the set of electoral systems (and thus voter incentives) that can be analyzed. In our case, the predicted behavior of voters is substantially different between plurality and AV.

The experiments reveal interesting patterns and support most of our theoretical predictions. We first study setups in which information is symmetric across states of nature. Under plurality, we observe the emergence of both types of equilibria: when the minority is sufficiently small, all groups stick to playing the informative equilibrium. By contrast,

\footnotetext{
${ }^{6}$ With three alternatives, plurality offers four possible actions: abstain, and vote for either one of the three alternatives. AV adds another four possible actions: three double approvals, and approving of all alternatives. Saari and Newenhizen (1988) argue that this may produce indeterminate outcomes, and Niemi (1984) argues that AV "begs voters to behave strategically", in a highly elaborate manner.
} 
when the minority is "large", in the sense that the informative equilibrium leads to a high probability of victory for the Condorcet Loser, all groups eventually decide to give up information aggregation and coordinate their ballots on the same alternative, as predicted by Duverger's Law. However, all groups begin by voting sincerely, and convergence to one of the two potential Duverger's Law equilibria is quite slow. Our theoretical model identifies a force explaining their behavior.

Under AV, and in line with theoretical predictions, some voters double vote to increase the vote shares of both majority candidates. As predicted, the amount of double voting also increases with the size of the minority. However, the absolute level of double voting is lower than predicted. Comparing the two systems, we observe that voters make fewer strategic mistakes under AV than under plurality. Moreover, when the minority is large, voters need more time to reach equilibrium play in plurality than in AV. This suggests that voters can more easily handle the larger set of voting possibilities offered by AV than the need to select an equilibrium under plurality.

In contrast with the theory (which focuses on symmetric equilibria), individual behavior displays substantial heterogeneity among subjects in AV: many subjects always double vote, whereas many other subjects always single vote their signal. The observation that double-voting increases with the size of the minority is mainly driven by a switch in the relative number of subjects in each cluster. This pattern points to the need to extend the theory and consider equilibria in asymmetric strategies. Extending the model in this direction, we find that this type of behavior is indeed an equilibrium which performs particularly well in explaining the level of double-voting observed in the laboratory.

We then turn to those treatments in which the quality of information varies across states of nature. In line with theoretical predictions, subjects eventually adjust their behavior to better aggregate information. In the case of plurality, the data provides further evidence to confirm our theoretical prediction that three-candidate equilibria are a natural focal point when majority voters have common values. In the case of AV, the results are even stronger, in the sense that voters converge faster toward the theoretical prediction.

Finally, we analyze the welfare properties of both electoral systems. A valuable feature of our common value setup among majority voters is that it allows us to make clear welfare 
predictions: in equilibrium, majority voters' payoff should be strictly higher with AV than in plurality. This is exactly what we observe in all different treatments.

\subsection{Related literature}

This paper relates to several strands of the literature on strategic voting and the comparison of electoral systems. We organize our discussion in three subsections: first, we review the literature on two-alternative elections in which voters have common values and private information about the quality of the two alternatives, i.e. there is an information aggregation problem. We then review the literature on multi-alternative elections with private valued voters, which focuses essentially on coordination problems. Lastly, we review the recent literature on multi-alternative elections with common values, in which our paper is rooted. This literature highlights the trade-off that majority voters face between aggregating information on the one hand and coordinating to defeat a weak candidate on the other. In all subsections, we review both the theoretical and experimental literature (we constrain ourselves to laboratory experiments).

\subsubsection{Two-Alternative Elections: Information Aggregation}

Theory The literature on the role of elections as a way of aggregating information dates back at least to Condorcet (1795). ${ }^{7}$ This desirable property of non-unanimous electoral systems is actually reinforced by the presence of strategic voters (Austen-Smith and Banks 1996, Feddersen and Pesendorfer 1996, 1997, 1999, Myerson 1998, and McMurray 2012).8,9 The robustness of this information aggregation property is discussed extensively in the literature. On the one hand, it is robust (to some extent) to the introduction of private values, i.e. all voters do not necessarily agree on the which alternative is best conditional on the state of nature (Gerardi 2000), costly voting (Krishna and Morgan 2011, 2012), and costly acquisition of information (Martinelli 2006, Oliveros 2011). On the other hand, it

\footnotetext{
${ }^{7}$ See Piketty (1999) for a review of the information-aggregation approach to political institutions.

${ }^{8}$ Two exceptions are Feddersen and Pesendorfer (1998), who show the inferiority of the unanimity rule, and Mandler (2012) who studies the case in which the probabilities to receive each signal are themselves random. Austen-Smith and Feddersen (2006) have shown that this conclusion is robust to pre-voting deliberations (on this topic, see also Coughlan 2001, and Eraslan and Bond 2009 ).

${ }^{9}$ Ladha et al. (1996) have identified situations in which there exists an asymmetric equilibrium in which voters who receive the same signal behave differently. This equilibrium leads to more efficient information aggregation than the sincere voting equilibrium in which voters vote their signal. Our section 6.3.1 about asymmetric equilibria in $\mathrm{AV}$ builds on that idea.
} 
is not robust to adversarial preferences (Fey and Kim 2007, Bhattacharya 2012), to voters preferring to vote for the winner (Callander 2008), and to voters being ambiguity averse (Ellis 2012).

Experiments The main message of the experimental literature on information aggregation in two-candidate elections is that subjects vote strategically and adapt to the different rules and parametrizations used in the experiments (although not to the extend predicted by theory). Guarnaschelli, McKelvey and Palfrey (2000) experimentally compare behavior under majority and unanimity rule. They find that, in a setting where information is symmetric across states, subjects vote sincerely when using majority but frequently vote against their signal when the rule in place is unanimity. Ladha, Miller and Oppenheimer (1996), Bhattacharya, Duffy and Kim (2012) and Bouton, Llorente-Saguer and Malherbe (2012) show that such strategic behavior is also present with majority rule in situations where information precision differs across states. ${ }^{10}$ Finally, Battaglini, Morton and Palfrey $(2008,2012)$ show that uninformed voters strategically abstain in order to delegate the decision to the informed ones, and even compensate for partisan biases. ${ }^{11}$

\subsubsection{Multi-Alternative Elections: Coordination Problems}

Theory Coordination problems are arguably the central issue in the literature on multialternative elections with private value voters. As shown repeatedly (see e.g. Myerson and Weber 1993, Cox 1997, Myerson 2000, 2002, Myatt 2007, Bouton 2012, Bouton and Gratton 2012), when a divided majority is facing a unified minority block, electoral systems produce (i) bad equilibria, in which the minority candidate gets elected, and (ii) equilibrium multiplicity, which leaves elections open to focal manipulations and coordination failures. In a purely private values environment, designing an electoral system exempt from such problems has so far proved impossible. ${ }^{12}$

The two systems under consideration in this paper, i.e. plurality and $\mathrm{AV}$, have been

\footnotetext{
${ }^{10}$ Goeree and Yariv (2010) provide further evidence that voters adjust their behavior to the electoral rule and show that pre-vote deliberations significantly diminishes institutional differences and uniformly improves efficiency.

${ }^{11}$ This feature has been also tested with observational data (McMurray 2012).

${ }^{12}$ Another interesting type of multi-alternative elections are those in which decisions on multiple binary issues are taken simultaneously, the so-called combinatorial voting. See Ahn and Oliveros (2012) for a thorough analysis.
} 
analyzed in private values environments. For plurality, the most famous result is the so-called Duverger's Law (Duverger 1963, Riker 1982, Palfrey 1989 and Cox 1997): "the simple-majority single-ballot system [the plurality electoral system] favors the two-party systems". Duverger's intuition is that voters have incentives to abandon their most preferred candidat if she has no chance to win the election to instead rally behind their most preferred serious candidate. In a setup including only strategic voters, Duverger's Law implies that only two candidates can obtain a positive fraction of the votes. Perhaps surprisingly, there is a least one equilibrium under plurality in which a Condorcet winner obtains zero vote. Myerson and Weber (1993) prove the existence of a non-Duverger's law equilibrium, in which three candidates obtain a positive fraction of the votes. Yet, as discussed by Fey (1997), the existence of such a non-Duverger's law equilibrium relies on quite demanding conditions on the structure of information in the electorate. Moreover, it is not expectationally stable. ${ }^{13,14}$ In contrast, in our setup, we prove the existence of an expectationally stable equilibrium in which three candidates receive a substantial fraction of the votes.

This strand of the literature also suggests that AV resists coordination problems better than many other electoral systems (Myerson and Weber 1993, Myerson 2000, 2002, Laslier 2010, Nunez 2010). In terms of voting behavior, the theoretical prediction is quite clear: there is no "hole" in the ballots cast by voters, i.e. voters identify a cutoff-candidate an only approve of the candidates yielding a utility at least as high as that one.

Experiments The experimental literature on multicandidate elections with private value voters is surprisingly small. ${ }^{15}$ The seminal papers of Forsythe et al $(1993,1996)$ are closest to our paper. They consider three-candidate elections in which a divided majority is opposed to a unified minority. Voters are perfectly informed about the distribution of types in the electorate. Forsythe et al (1993) finds that, in elections without polls or shared history, plurality rule frequently leads to a victory of the Condorcet loser. However, both polls and shared histories (i) decrease the frequency of such coordination failure among

\footnotetext{
${ }^{13}$ This non-Duverger's Law equilibrium might also be deemed conterintuitive since it requires the supporters of the strongest majority candidate mix between their most-preferred candidate and the other majority candidate.

${ }^{14}$ See Myatt (2007) for a discussion of the existence of non-Duverger's law equilibria in a setup with aggregate uncertainty.

${ }^{15}$ See Rietz (2008), Laslier (2010) or Palfrey (2012) for more detailed reviews of that literature.
} 
majority voters, and (ii) favor the emergence of Duverger's law. ${ }^{16}$ Forsythe et al. (1996) analyze alternative voting procedures. They find that, under AV, it is easier for majority voters to overcome the coordination problem. Granic (2012) successfully replicates these findings and extends them to settings where subjects are imperfectly informed about the distribution of types in the electorate. ${ }^{17}$

\subsubsection{Multi-Alternative Elections: Information Aggregation and Coordination Problems}

Theory Few papers analyzing multi-alternative elections with common valued voters. This strand of the literature is divided into two parts: analyses considering (i) setups with pure common values voters, and (ii) setups with both private and common values voters. McLennan (1998) shows that, in games of pure common values, any strategy that maximizes utility is an equilibrium. Ahn and Oliveros (2011a) exploit this to prove that in a pure common value setup, "the maximal equilibrium utility under approval voting is greater than or equal to the maximal equilibrium utility under plurality voting or under negative voting." (p. 3). ${ }^{18}$

Analyses including both private and common values voters capture an essential tradeoff between two goals in multi-alternative elections: aggregating information on the one hand and coordinating against a weak candidate on the other. ${ }^{19}$ Goertz and Maniquet (2011) find a numerical example in which approval voting fails to aggregate information. That example crucially relies on a large fraction of the voters having assigning a zeroprobability to one state of nature. Bouton and Castanheira (2012) show that, in large elections, AV satisfies Full Information and Coordination Equivalence. That is, it solves both the information aggregation problem and the coordination problem at once. A nec-

\footnotetext{
${ }^{16}$ Their findings are consistent with later evidence on other setups by Gerber et al. (1998), Bassi (2008) or Hizen et al (2010).

${ }^{17}$ Van der Straeten et al. (2010) or Bassi (2008) also study AV experimentally although in substantially different settings. Van der Straeten et al. (2010) show that in a setting where alternatives can be ordered in a one dimensional policy line, voters are strategic and the Condorcet winner wins substantially more compared to plurality, runoff elections or single transferale vote. Bassi (2008) shows that strategic voting is substantial in $\mathrm{AV}$, but less than with plurality and more than with Borda rule.

${ }^{18}$ As mentioned above, another interesting type of multi-alternative elections are those in which decisions on multiple binary issues are taken simultaneously, the so-called combinatorial voting. Ahn and Oliveros (2011b) analyze such elections in a pure common value setup.

${ }^{19}$ In such a setup, Piketty (2000) analyzes the incentives of voters to communicate between two different elections or between the two rounds of a majority runoff election (see also Castanheira 2003). See Martinelli (2002) for a more detailed analysis of information aggregation in three-candidate majority runoff elections.
} 
essary and sufficient condition for this result is that sufficiently many voters may need additional information to identify their best alternative (i.e. they have a "doubt" that information can dispel).

From a theory standpoint, the paper closest to ours is Bouton and Castanheira (2012). There are several important differences between their model and ours. The most important is that instead of focusing on limiting properties when the electorate grows large, we consider electorates of any size. Relaxing this assumption does not come at no cost: we are indeed forced to focus on a more stylized structure of preferences. For instance, we cannot analyze situations in which majority voters have heterogenous preferences (and may not agree on which candidate is best when information is perfect). Moreover, we can neither provide a general proof of uniqueness, nor determine the equilibrium strategy in all cases. Yet, as highlighted in the introduction, we prove several novel results that we then bring to the laboratory.

Experiments To the best of our knowledge, our paper is the first laboratory experiment which explores multi-alternative elections with common value voters.

\section{A common value model}

We consider a voting game with an electorate of fixed and finite size who must elect a policy $P$ out of three possible alternatives, $A, B$ and $C$. The electorate is split in two groups: $n$

active voters who constitute a majority, and $n_{C}$ voters who constitute a minority. There are two states of nature: $\omega=\{a, b\}$, which materialize with probabilities $q(\omega)>0$. While these probabilities are common knowledge, the actual state of nature is not observed by the time of the election.

Active voters' utility depends both on the policy outcome and on the state of nature: utility is high $(U=V)$ if $A$ is elected and the state is $a$, or if $B$ is elected and the state is $b$. It is intermediate $(U=v \in(0, V))$ if $A$ wins and the state is $b$ or if $B$ wins and the 
state is $a$. Finally, utility is low (normalized to zero) if $C$ is elected:

$$
\begin{aligned}
U(P \mid \omega) & =V \text { if }(P, \omega)=(A, a) \text { or }(B, b) \\
& =v \text { if }(P, \omega)=(A, b) \text { or }(B, a) \\
& =0 \text { if } P=C .
\end{aligned}
$$

For the sake of simplicity, minority voters are passive in the game: they always vote for $C$, which means that $C$ receives $n_{C}$ ballots independently of the state of nature or the electoral system. This implies that active voters must cast at least $n_{C}$ ballots in favor of either $A$ or $B$ to avoid the victory of $C$. We focus on the interesting case in which $C$-voters represent a large minority: $n-1>n_{C}>n / 2$. Hence, $C$ is a Condorcet loser (it would lose against either $A$ or $B$ in a one-on-one contest), but it can win the election if active voters split their votes between $A$ and $B$.

Timing. Before the election (at time 0), nature chooses whether the state is $a$ or $b$. At time 1, each voter receives a signal $s \in\left\{s_{A}, s_{B}\right\}$, with conditional probabilities $r(s \mid \omega)>0$ and $r\left(s_{A} \mid \omega\right)+r\left(s_{B} \mid \omega\right)=1$. Probabilities are common knowledge but signals are private. We set signal $s_{A}$ to be more likely in state $a$ than in state $b$ :

$$
\begin{aligned}
& r\left(s_{A} \mid a\right)>r\left(s_{A} \mid b\right), \text { and therefore } \\
& r\left(s_{B} \mid a\right)<r\left(s_{B} \mid b\right) .
\end{aligned}
$$

The distribution of signals is unbiased if $r\left(s_{A} \mid a\right)=r\left(s_{B} \mid b\right)$. Note that $r\left(s_{A} \mid a\right)+r\left(s_{A} \mid b\right)=$ 1 in this case. The distribution of signals is biased if $r\left(s_{A} \mid a\right) \neq r\left(s_{B} \mid b\right)$ and, by convention, we will focus on the case in which the "more abundant" signal is $s_{A}: r\left(s_{A} \mid a\right)+r\left(s_{A} \mid b\right)>1$.

Having received her signal, the voter updates her beliefs about each state through Bayes' rule, to:

$$
q(\omega \mid s)=\frac{q(\omega) r(s \mid \omega)}{q(a) r(s \mid a)+q(b) r(s \mid b)} .
$$

Like Bouton and Castanheira (2012), we assume that signals are sufficiently strong to create a divided majority:

$$
\frac{q\left(a \mid s_{A}\right)}{q\left(b \mid s_{A}\right)}>1>\frac{q\left(a \mid s_{B}\right)}{q\left(b \mid s_{B}\right)} .
$$


That is, conditional on receiving signal $s_{A}$, alternative $A$ yields strictly higher expected utility than alternative $B$, and conversely for a voter who receives signal $s_{B}$.

The election is held at time $\mathbf{2}$, when the actual state of nature is still unobserved, and payoffs realize at time 3: the winner of the election and the actual state of nature are revealed, and each voter receives her utility $U(P, \omega)$.

Strategy space and equilibrium concept. The alternative winning the election is the one receiving the largest number of votes, with ties being broken by a fair dice. Still, the action space, i.e. which ballots are feasible, depends on the electoral rule. We consider two such rules: plurality and approval voting.

In plurality, each voter can cast a ballot on one alternative or abstain. The voters' action set is thus:

$$
\Psi_{P l u}=\{A, B, C, \varnothing\}
$$

where, by an abuse of notation, action $A$ (respectively $B, C$ ) denotes a ballot in favor of $A$ (resp. $B, C$ ) and $\varnothing$ denotes abstention. ${ }^{20}$

In approval voting, each voter can approve of as many (or as few) alternatives as she wishes:

$$
\Psi_{A V}=\{A, B, C, A B, A C, B C, A B C, \varnothing\}
$$

where, by an abuse of notation, action $A$ denotes a ballot in favor of $A$ only, action $B C$ denotes a joint approval of $B$ and $C$, etc. Each approval counts as one vote: when a voter only approves of $A$, then only alternative $A$ is credited with a vote. If the voter approves of both $A$ and $B$, then $A$ and $B$ are credited with one vote each, and so on. As in plurality, the alternative with the most votes wins the election.

The only difference between approval voting and plurality is that a voter can also cast a double or triple approval. While a single approval $(\psi=A, B$ and $C)$ can be pivotal against any other alternative, double approvals $(\psi=A B, B C$ and $A C)$ can only be pivotal against one precise alternative. For instance, if the voter plays $A C$, she is voting against $B$ : her ballot can only be pivotal against that alternative, either in favor of $A$ or of $C$. Finally, a triple approval $(A B C)$ can never be pivotal: it is strategically equivalent to abstention.

\footnotetext{
${ }^{20}$ Abstention will turn out to be a dominated action in both rules. Hence, removing abstention from the choice set would not affect the analysis.
} 
Let $x_{\psi}$ denote the number of voters who played action $\psi \in \Psi$ at time 2 . The total number of votes received by an alternative $\psi$ is denoted by $X_{\psi}$. Under plurality the total number of votes received by alternative $A$, for instance, is simply: $X_{A}=x_{A}$. Under approval voting, it is: $X_{A}=x_{A}+x_{A B}+x_{A C}+x_{A B C}$.

A symmetric strategy is a mapping $\sigma: S \rightarrow \triangle\left(\Psi_{R}\right)$, where $\sigma_{s}(\psi)$ denotes the probability that some randomly sampled voter who received signal $s$ plays action $\psi, S$ is the set of possible signals, and $\Delta\left(\Psi_{R}\right)$ is the simplex over the actions possible under the electoral rule $R \in\{P L U, A V\}$. Given a strategy $\sigma$, the expected share of voters playing action $\psi$ in state $\omega$ is thus:

$$
\tau_{\psi}^{\omega}(\sigma)=\sum_{s} \sigma_{s}(\psi) \times r(s \mid \omega)
$$

The expected number of ballots $\psi$ is:

$$
\mathrm{E}[x(\psi) \mid \omega, \sigma]=\tau_{\psi}^{\omega}(\sigma) \times n .
$$

Since voters do not observe the state of nature, expected vote shares only vary across states because the expected fraction of active voters receiving each signal is different.

Let an action profile $x$ be the vector that lists, for each action $\psi$, the realized number of ballots $\psi$. Since we focus on symmetric strategies for the time being, and since the conditional probabilities of receiving a signal $s$ are iid, the probability distribution over the possible action profiles is given by the multinomial probability distribution:

$$
\operatorname{Pr}(x \mid \omega)=n ! \prod_{\psi \in \Psi_{R}} \frac{\tau_{\psi}^{\omega}(\sigma)^{x(\psi)}}{x(\psi) !}, R=P l u, A V
$$

For this voting game, we analyze the properties of Bayesian Nash equilibria that (1) do not involve weakly dominated strategies and (2) satisfy what we call sincere stability. That is, we impose the refinement that the equilibrium must be robust to the presence of an arbitrarily small fraction $\varepsilon$ of sincere votes (that is: $\sigma_{s_{A}}(A), \sigma_{s_{B}}(B) \geq \varepsilon>0$ ), and we look for sequences of equilibria with $\varepsilon \rightarrow 0$.

The reason why we introduce sincere stability is to get rid of equilibria that would only be sustainable when all pivot probabilities are exactly zero, and all voters are then indifferent between all actions. Imagine for instance that all active voters play $A$. In that 
case, the number of votes for $A$ is $n$ and the number of votes for $C$ is $n_{C}$, with probability 1. Voters are then indifferent between all possible actions, since a ballot can never be pivotal. In contrast, sincere stability imposes that a small fraction of the voters votes for their preferred alternative. This implies that at least some pivot probabilities become strictly positive, and hence that indifference is broken.

There are two main arguments in favor of our sincere stability refinement. First, it captures the essence of properness in a very tractable way. ${ }^{21},{ }^{22}$ Second, it is behaviorally relevant since experimental data (both in our experiments and others) suggest that some voters vote for their ex ante most preferred alternative no matter what.

\section{Plurality}

This section analyzes the equilibrium properties of plurality voting, in which voters can either vote for one alternative or abstain. We find that two types of equilibria coexist: in one, all active voters play a same (pure) strategy independently of their signal: they all vote either for $A$ or for $B$. This type of equilibrium is known as a Duverger's Law equilibrium, in which only two alternatives receive a strictly positive vote share (Palfrey 1989, Cox 1997). In the second type of equilibrium, an active voter's strategy does depend on her signal. Depending on parameter values, this equilibrium either features sincere voting, that is voters with signal $s_{A}$ (resp. $s_{B}$ ) vote $A$ (resp. $B$ ) or a strictly mixed strategy in which voters with the most abundant signal ( $s_{A}$ by convention) mix between $A$ and $B$. Importantly, these three-party equilibria exist for any population size and are robust to signal biases. In other words, they do not rely on $A$ and $B$ 's vote shares being identical. To the contrary: they imply that vote shares differ.

\subsection{Pivot Probabilities and Payoffs}

When deciding for which alternative to vote, a voter must first assess the expected value of each possible ballot. This value depends on the possible pivot events: unless the ballot

\footnotetext{
${ }^{21}$ We are grateful to Eric Van Damme for insightful discussions about refinement concepts in voting games.

${ }^{22}$ We do not use more traditional refinement concepts such as perfection or properness because, in the voting context, the former does not have much bite since weakly dominated strategies are typically excluded from the equilibrium analysis. The latter is less tractable since it requires a sophisticated comparison of pivot probabilities for totally mixed strategies.
} 
is pivotal and affects the outcome of the election, it leaves the voter's utility unchanged. We denote by piv $_{Q P}$ the pivot event that one voter's ballot changes the outcome of the election from a victory of $P$ towards a victory of $Q$.

In our setup, the comparison between the three potentially relevant actions, $A, B$ and $C$, is simplified by two elements: first, voting for $C$ is a dominated action. Hence, we can set $\tau_{C}^{\omega}(\sigma)$ equal to zero. Second, a vote for $A$ or for $B$ can only be pivotal against $C$, since we impose that $n_{C}>n / 2$. This implies that abstention is also a dominated action, and simplifies the other computations without affecting generality.

Voter $i$ 's ballot can only be pivotal, say in favour of $A$ if, without $i$ 's ballot, the number of $A$-ballots $\left(x_{A}\right)$ is either the same as or one less than the number of $C$-ballots, $n_{C}$, and $i$ votes for $A$. To assess the probability of such an event, each active voter must identify the distribution of the other $n-1$ voters' ballots, given the strategy $\sigma$. Dropping $\sigma$ from the vote shares $\tau_{\psi}^{\omega}$ for the sake or readability, the probability of one vote being pivotal, respectively in favour of $A$ and $B$ is:

$$
\begin{aligned}
& p_{A C}^{\omega} \equiv \operatorname{Pr}\left(\text { piv }_{A C} \mid \omega, \text { Plurality }\right)=\frac{(n-1) !}{2} \frac{\left(\tau_{A}^{\omega}\right)^{n-1}\left(\tau_{B}^{\omega}\right)^{n-n_{C}-1}}{\left(n_{C}-1\right) !\left(n-n_{C}-1\right) !}\left[\frac{\tau_{A}^{\omega}}{n_{C}}+\frac{\tau_{B}^{\omega}}{n-n_{C}}\right] \\
& p_{B C}^{\omega} \equiv \operatorname{Pr}\left(\text { piv }_{B C} \mid \omega, \text { Plurality }\right)=\frac{(n-1) !}{2} \frac{\left(\tau_{B}^{\omega}\right)^{n_{C}-1}\left(\tau_{A}^{\omega}\right)^{n-n_{C}-1}}{\left(n_{C}-1\right) !\left(n-n_{C}-1\right) !}\left[\frac{\tau_{B}^{\omega}}{n_{C}}+\frac{\tau_{A}^{\omega}}{n-n_{C}}\right]
\end{aligned}
$$

where the two terms between brackets represent the cases in which one breaks and makes a tie. Note that the pivot probabilities $p_{A C}^{\omega}$ and $p_{B C}^{\omega}$ are continuous in $\tau_{A}^{\omega}$ and $\tau_{B}^{\omega}$.

Let $G(\psi \mid s)$ denote the expected gain of an action $\psi \in\{A, B\}$ over abstention, $\varnothing$ :

$$
\begin{aligned}
& G(A \mid s)=q(a \mid s) p_{A C}^{a} V+q(b \mid s) p_{A C}^{b} v(>0) \\
& G(B \mid s)=q(a \mid s) p_{B C}^{a} v+q(b \mid s) p_{B C}^{b} V(>0) .
\end{aligned}
$$

It is obvious that both actions yield higher payoffs than abstention, which is thus dominated. The pay-off difference between actions $A$ and $B$ is:

$$
G(A \mid s)-G(B \mid s)=q(a \mid s)\left[V p_{A C}^{a}-v p_{B C}^{a}\right]+q(b \mid s)\left[v p_{A C}^{b}-V p_{B C}^{b}\right]
$$




\subsection{Duverger's Law Equilibria}

The game theoretic version of Duverger's Law (Duverger 1963) states that, in plurality elections, only two alternatives should obtain a strictly positive fraction of the votes when voters play strategically (Palfrey 1989, Myerson and Weber 1993, Bouton and Castanheira 2009):

Definition 1 A Duverger's Law equilibrium is a voting equilibrium in which only two alternatives obtain a strictly positive fraction of the votes.

Our first proposition shows that:

Proposition 1 In plurality, Duverger's law equilibria exist for any electorate size, prior probabilities of the two states, and distribution of signals.

Proof. Consider $\sigma_{s_{A}}(A)=\varepsilon$ and $\sigma_{s_{B}}(B)=1$. From (5) and (6), we have:

$$
\frac{p_{A C}^{\omega}}{p_{B C}^{\omega}}=\left(\frac{\tau_{A}^{\omega}}{\tau_{B}^{\omega}}\right)^{2 n_{C}-n} \frac{\tau_{A}^{\omega}\left(n-n_{C}\right)+\tau_{B}^{\omega} n_{C}}{\tau_{A}^{\omega} n_{C}+\tau_{B}^{\omega}\left(n-n_{C}\right)} \underset{\varepsilon \rightarrow 0}{\rightarrow} 0
$$

Hence, from (9), we have that $G(A \mid s)-G(B \mid s)<0$ for any $\varepsilon$ in the neighborhood of 0 .

These equilibria are such that either all active voters vote for alternative $A$ or they all vote for alternative $B$. Duverger's Law equilibria feature pros and cons. On the one hand, they ensure that either $A$ or $B$ receives strictly more votes than $C$, and hence that $C$ cannot win the election. On the other hand, they prevent learning. That is, the winner of the election cannot vary with the state of nature.

The reason why Duverger's Law equilibria exist in plurality elections is the classical one: voters do not want to waste their ballot on an alternative that is very unlikely to win. Consider for instance the strategy profile $\sigma\left(B \mid s_{A}\right)=1-\varepsilon$ and $\sigma\left(B \mid s_{B}\right)=1$ with $\varepsilon$ strictly positive but arbitrarily small. In that case, an $A$-ballot is much less likely to be pivotal against $C$ than a $B$-ballot. ${ }^{23}$ Therefore, the value of a $B$-ballot is larger than that of an $A$-ballot, both for $s_{A^{-}}$and $s_{B}$-voters.

\footnotetext{
${ }^{23}$ For $\sigma\left(B \mid s_{A}\right)=1=\sigma\left(B \mid s_{B}\right)$, all pivot probabilities are equal to zero. In this case, voters are indifferent between all actions. Sincere stability means that we identify incentives for $\sigma\left(B \mid s_{A}\right) \rightarrow 1$. They imply that $G\left(B \mid s_{A}\right)>G\left(A \mid s_{A}\right)$ in the neighborhood of this Duverger's Law equilibrium.
} 


\subsection{Informative Equilibria}

In Duverger's Law equilibria, all active voters play the same (pure) strategy independently of their signal. Information about voter preferences is therefore lost. Still, this type of equilibrium is typically considered as the only reasonable one if voters are short-term instrumentally rational, in Cox's (1997) terminology. Indeed, in a classical private value setup, equilibria violating Duverger's Law require that the vote shares of the second and third alternatives are (almost) equal, a "knife-edge" case (Palfrey 1989). Therefore, empirical research associates strategic voting with the propensity for a voter to abandon his or her most-preferred candidate in order to vote for a second-best candidate who is a more serious contender (see Blais and Nadeau 1996, Cox 1997, Alvarez and Nagler 2000, Blais et al. 2005). Observing that only relatively low fractions of the electorate switch to their second-best alternative in this way is thus interpreted as evidence that few voters are instrumental or rational.

Yet, as shown by Propositions 2 and 3 below, common values among majority voters give rise to other equilibria in which "short-term instrumentally rational voters" should actually deviate from either Duverger's Law equilibria or "knife-edge" three-candidate equilibria. The key difference is that, in our setup, voters value the information generated by their own and by other voters' ballots. Like in Austen-Smith and Banks (1996) and Myerson (1998), they compare their probability of being pivotal in each state of nature. In what we call an informative equilibrium, voting strategies imply that these pivot probabilities are sufficiently close to one another. Such an equilibrium entails that (a) all alternatives receive a strictly positive vote share, (b) in a given state, the expected vote shares of each alternative are different, and (c) $A$ is the strongest majority contender in one state of nature, and $B$ in the other state. ${ }^{24}$

When information is close to being symmetric across states, voters vote sincerely in an informative equilibrium: a voter who receives signal $s_{A}$ then votes for $A$, whereas a voter who receives signal $s_{B}$ votes for $B$. That is, abandoning one's preferred candidate would not be a best response when one expects other voters to vote sincerely:

\footnotetext{
${ }^{24}$ If, in addition, the expected vote share of $A$ and of $B$ in their respective state is sufficiently larger than $C$ 's, then the informative equilibrium is also expectationally stable in the sense of Fey (1997). See also Bouton and Castanheira (2009, Propositions 7.3 and 7.4).
} 
Proposition 2 In the unbiased case $r\left(s_{A} \mid a\right)=r\left(s_{B} \mid b\right)$, the sincere voting equilibrium exists $\forall n, n_{c}$. Moreover, there exists a value $\delta\left(n, n_{c}\right)>0$ such that sincere voting is an equilibrium for any asymmetric distributions satisfying $r\left(s_{A} \mid a\right)-r\left(s_{B} \mid b\right)<\delta\left(n, n_{c}\right)$.

Proof. We start with the symmetric case, i.e. $r\left(s_{A} \mid a\right)=r\left(s_{B} \mid b\right)$. Under sincere voting, $\sigma_{s_{A}}(A)=1=\sigma_{s_{B}}(B)$ (note that sincere stability is not a binding condition in this case), (5) and (6) imply $p_{A C}^{a}=p_{B C}^{b}>p_{A C}^{b}=p_{B C}^{a}$. Then, from (9):

$$
G(A \mid s)-G(B \mid s)=\left[V p_{A C}^{a}-v p_{B C}^{a}\right][q(a \mid s)-q(b \mid s)]
$$

Since $q\left(a \mid s_{A}\right)-q\left(b \mid s_{A}\right)>0>q\left(a \mid s_{B}\right)-q\left(b \mid s_{B}\right)$, this implies $G\left(A \mid s_{A}\right)-G\left(B \mid s_{A}\right)>0>$ $G\left(A \mid s_{B}\right)-G\left(B \mid s_{B}\right)$. Sincere voting is thus an equilibrium strategy. By the continuity of pivot probabilities with respect to $\tau_{A}^{\omega}$ and $\tau_{B}^{\omega}$, it immediately follows that there must exist a value $\delta\left(n, n_{C}\right)>0$ such that sincere voting is an equilibrium for any $\left|r\left(s_{A} \mid a\right)-r\left(s_{B} \mid b\right)\right|<\delta\left(n, n_{c}\right)$.

The intuition for the proof is simply that, in the unbiased case, sincere voting implies that the likelihood of being pivotal against $C$ is the same with an $A$-ballot in state $a$ as with a $B$-ballot in state $b$. Therefore, $s_{A}$-voters strictly prefer to vote for $A$ and $s_{B}$-voters strictly prefer to vote for $B$. The pros and cons of sincere voting are the exact flipside of the ones identified for Duverger's Law equilibria: as illustrated by the following example, ${ }^{25}$ it allows for learning, but does not guarantee the defeat of the Condorcet loser.

Example 1 Consider a case in which $n=12, n_{C}=7$, and $r\left(s_{A} \mid a\right)=r\left(s_{B} \mid b\right)=2 / 3$. In this case, sincere voting implies that the best alternative ( $A$ in state a or $B$ in state b) has the highest expected vote share and wins with a probability of $73 \%$. C then has the second largest expected vote share and wins with a probability of $23 \%$ in either state. The alternative with the lowest -but strictly positive-vote share is $B$ in state a and $A$ in state $b$.

When $n_{C}$ is 9, the alternative with the largest expected vote share is $C$. In this informative equilibrium, $C$ thus wins with a probability above $71 \%$, whereas the best alternative wins with a probability below $29 \%$.

Based on Proposition 2 and Example 1, one may be misled into thinking that infor-

\footnotetext{
${ }^{25}$ Each numerical example reproduces the parameters used in one of the treatments of our laboratory experiments (see section 5). In all examples, the payoffs are: $V=200 ; v=110$ and the value of $C$ is 20 . Normalizing the latter to 0 would also reduce the other payoffs by 20 .
} 
mative equilibria require signals to be unbiased. Two remarks are in order. First, voting sincerely is still an equilibrium when the signal bias exists but is not too large. In that case, the difference in beliefs between $s_{A}$ and $s_{B}$-voters keeps dominating the difference in pivot probabilities. Yet, for any given bias $r\left(s_{A} \mid a\right)-r\left(s_{B} \mid b\right)>0$, sincere voting is only an equilibrium if electorate size is sufficiently small: as electorate size increases to infinity, given the biased signal structure, the ratio of pivot probabilities would either converge to zero or infinity if voters kept voting sincerely.

Second, the fact that the signal structure becomes too biased to sustain sincere voting does not imply that voters switch to a Duverger's Law equilibrium: Proposition 3 instead shows that, in an informative equilibrium, $s_{A}$-voters adopt a mixed strategy and vote for $B$ with strictly positive probability. This allows them to lean against the bias in the signal structure. In other words, the short-term instrumentally rational voter should partly abandon the strongest contender and lend support to the weakest majority alternative:

Proposition 3 Let $r\left(s_{A} \mid a\right)-r\left(s_{B} \mid b\right)>\delta\left(n, n_{c}\right)$. Then, there exists a mixed strategy equilibrium with $\sigma_{s_{A}}(A) \in(0,1)$ and $\sigma_{s_{B}}(B)=1$, such that alternative $A$ receives strictly more votes in state a than in state $b$, and conversely for alternative $B$.

Proof. See Appendix A2.

The intuition for this result is that strong biases in the signal structure imply that the difference in pivot probabilities between states $a$ and $b$ becomes too large if voters were to vote sincerely. To correct for this bias in the informative equilibrium, $s_{A}$-voters must strictly mix between $A$ and $B$. In this way, they partially compensate the gap in pivot probabilities caused by the bias. The proof of the proposition establishes that there always exists one such mixture that is an equilibrium. It is such that $s_{A^{-}}$-voters are indifferent between voting $A$ and $B$, whereas $s_{B}$-voters strictly prefer the latter. The intuition for the proof of this result is best conveyed with the help of a second example:

Example 2 Electorate size is $n=12$ and $n_{C}=7$, and the signal structure is $r\left(s_{A} \mid a\right)=$ $8 / 9>2 / 3=r\left(s_{B} \mid b\right)$. The two states of nature are equally likely: $q(\omega)=1 / 2$. For these parameter values, an $s_{A}$-voter would prefer to vote for $B$ if all the other voters were to 
vote sincerely. Indeed, sincere voting implies: ${ }^{26}$

$$
\frac{q\left(a \mid s_{A}\right)}{q\left(b \mid s_{A}\right)}=\frac{8}{11}<13.5=\frac{V p_{B C}^{b}-v p_{A C}^{b}}{V p_{A C}^{a}-v p_{B C}^{a}} .
$$

That is, the weighted probabilities of being pivotal in favour of $B$ in state $b$ is much larger than the pivot probabilities in favour of $A$, which implies $G\left(A \mid s_{A}\right)-G\left(B \mid s_{A}\right)<0$. The mixed-strategy equilibrium is reached when $\sigma_{s_{A}}(A)=0.9153$ and $\sigma_{s_{B}}(B)=1$ : by reducing the expected vote share of $A$ and increasing the vote share of $B$, the relative probability of being pivotal in favour of $A$ in state a has increased to the point in which:

$$
\frac{q\left(a \mid s_{A}\right)}{q\left(b \mid s_{A}\right)}=\frac{8}{11}=\frac{V p_{B C}^{b}-v p_{A C}^{b}}{V p_{A C}^{a}-v p_{B C}^{a}}>\frac{q\left(a \mid s_{B}\right)}{q\left(b \mid s_{B}\right)}=\frac{1}{3} .
$$

Expressed in terms of payoffs, this means that $s_{A}$-voters are now indifferent between voting $A$ and $B$, whereas $s_{B}$-voters strictly prefer to vote B.Also interesting is that this equilibrium shares relevant features with a Condorcet-Jury type of equilibrium: even though vote shares are substantially different across states and alternatives:

$$
\tau_{A}^{a}=0.81>\tau_{B}^{b}=0.69>\frac{n_{C}}{n}=0.58>\tau_{A}^{b}=0.31>\tau_{B}^{a}=0.19
$$

$A$ leads in state $a$ and $B$ leads in state $b$. Their winning probabilities are respectively $96 \%$ and $79 \%$. Yet, this informative equilibrium gives $C$ a strictly positive probability of victory (18\% in state $b$ and $3 \%$ in state a). Importantly, though, expected utility is higher in this equilibrium than in Duverger's Law equilibria.

The example vividly illustrates that neither the existence nor the stability of this equilibrium relies on some form of symmetry between vote shares. Also, as proved by Bouton and Castanheira (2009) for large Poisson games, this mixed-strategy equilibrium also exists in very large electorates (i.e. for $n \rightarrow \infty$ ), with the difference that the gap between $\tau_{A}^{a}$ and $\tau_{B}^{b}$ decreases to zero (i.e. $\lim _{n \rightarrow \infty} \tau_{A}^{a}=\lim _{n \rightarrow \infty} \tau_{B}^{b}$ ), and that stability relies on $r\left(s_{A} \mid a\right)$ being sufficiently larger than $n_{C} / n$.

\footnotetext{
${ }^{26}$ Note that, by $(9), G(A \mid s)-G(B \mid s)>0$ iff the left-hand side of (10) is larger than the right-hand side.
} 


\section{Approval Voting}

\subsection{Payoffs and Dominated Strategies}

Under approval voting, voters have access to a larger choice set, which makes their choice potentially more complex. Single approvals $(A, B, C)$ have exactly the same effect as in plurality. Double or triple approvals instead ensure that one selectively abstains between the approved alternatives. For instance, an $A B$-ballot can only be pivotal against $C$. The following lemma shows that the set of undominated strategies is more restricted:

Lemma 1 Independently of a voter's signal, the actions $\psi \in\{C, A C, B C, A B C, \varnothing\}$ are weakly dominated by some action in $\psi \in\{A, B, A B\}$. Hence, in equilibrium:

$$
\sigma_{s}(A)+\sigma_{s}(B)+\sigma_{s}(A B)=1, \forall s \in\left\{s_{A}, s_{B}\right\}
$$

Proof. The proof is straightforward: consider a majority-block voter and compare actions $A B$ and $A B C$. While the latter can never be pivotal, an $A B$-ballot can be pivotal against $C$, either in favor of $A$ or in favor of $B$. In both cases, and independently of the true state of nature, utility can only increase. Hence, $A B$ weakly dominates $A B C$. All other dominance relationships are obtained by performing similar two-by-two comparisons: $A B$ weakly dominates $A B C, \varnothing$ and $C ; A$ weakly dominates $A C$; and $B$ weakly dominates $B C$.

The intuition for the lemma is that abstaining or approving of $C$ can only increase $C$ 's probability of winning. In contrast, the actions in the undominated set $(A, B$, and $A B)$ can only reduce it. The remaining question is how a voter may want to allocate her ballot across these undominated actions. This depends on the value of each undominated action, which itself depends on the pivot events. Let $\pi_{Q P}^{\omega}$ denote the probability that a single- $Q$ ballot by some voter $i$ is pivotal in favor of $Q$ at the expense of $P$ in state of nature $\omega \in\{a, b\}$ and the voting rule is approval voting. The precise derivation of these pivot probabilities is detailed in Appendix A1.

For a voter who received signal $s$, the value $G^{A V}$ of a single- $A$ ballot under approval voting is then:

$$
G^{A V}(A \mid s)=q(a \mid s)\left[\pi_{A C}^{a} V+\pi_{A B}^{a}(V-v)\right]+q(b \mid s)\left[\pi_{A C}^{b} v+\pi_{A B}^{b}(v-V)\right] .
$$


Note that the probability of being pivotal between $A$ and $B$ is no longer zero. This is due to the possibility of double voting, which can increase the total score of both $A$ and $B$ above that of $C$. Similarly, the value of a single- $B$ ballot is:

$$
G^{A V}(B \mid s)=q(a \mid s)\left[\pi_{B C}^{a} v+\pi_{B A}^{a}(v-V)\right]+q(b \mid s)\left[\pi_{B C}^{b} V+\pi_{B A}^{b}(V-v)\right]
$$

The value of a double ballot follows almost immediately from (12) and (13). Double voting cannot be pivotal between $A$ and $B$, while adding up the chances of being pivotal against $C$, either in favor of $A$ or in favor of $B$ :

$$
G^{A V}(A B \mid s)=q(a \mid s)\left[\pi_{A C}^{a} V+\pi_{B C}^{a} v-\phi^{a}\right]+q(b \mid s)\left[\pi_{A C}^{b} v+\pi_{B C}^{b} V-\phi^{b}\right],
$$

where $\phi^{a}$ and $\phi^{b}$ are correcting factors for three-way ties (see Appendix A1 for a precise definition). Consider the case in which, without voter $i$ 's ballot, both alternatives $A$ and $B$ lose to $C$ by one vote (that is, both obtain $n_{C}-1$ votes). A single- $A$ ballot creates a tie between $A$ and $C$. Thus, the ballot is pivotal in favor of $A$ with probability $1 / 2$. Likewise, a single- $B$ ballot is pivotal in favor of $B$ with probability $1 / 2$. Yet, a double vote $A B$ creates a three-way tie, which still allows $C$ to win with probability $1 / 3$ : the winning probabilities of $A$ and $B$ are $1 / 3$ instead of $1 / 2$. Summing up the probabilities $\pi_{A C}^{a}$ and $\pi_{B C}^{a}$ thus overestimates the value of the double ballot by $(V+v) / 6$. The terms $\phi^{a}$ and $\phi^{b}$ correct for these overestimations in that and three other cases: when $A$ trails behind both $B$ and $C$ by one vote, when $B$ trails behind both $A$ and $C$ by one vote, and when $A, B$ and $C$ have the same number of votes. These correcting factors become vanishingly small when the population size increases towards infinity, because the probability of three-way ties also becomes vanishingly small compared to other two-way pivot events. Yet, our purpose in this paper is to assess the properties of plurality and approval voting both for small-committee and for large-population elections, which implies that we need to take account of such three-way ties. ${ }^{27}$

\footnotetext{
${ }^{27}$ These correcting factors actually prove extremely relevant in small committees, in particular for the characterization of the asymmetric equilibria that we analyze in Section 6.3.1.
} 
The payoff differential between actions $A$ and $A B$ is therefore:

$$
\begin{aligned}
G^{A V}(A \mid s)-G^{A V}(A B \mid s)= & q(a \mid s)\left[\pi_{A B}^{a}(V-v)-\pi_{B C}^{a} v+\phi^{a}\right] \\
& +q(b \mid s)\left[\pi_{A B}^{b}(v-V)-\pi_{B C}^{b} V+\phi^{b}\right] .
\end{aligned}
$$

With straightforward, although tedious, manipulations, one finds that the first term in (15) may either be positive or negative, whereas the second is strictly negative. Similarly, the first term in (16) must be strictly negative:

$$
\begin{aligned}
G^{A V}(B \mid s)-G^{A V}(A B \mid s)= & q(a \mid s)\left[\pi_{B A}^{a}(v-V)-\pi_{A C}^{a} V+\phi^{a}\right] \\
& +q(b \mid s)\left[\pi_{B A}^{b}(V-v)-\pi_{A C}^{b} v+\phi^{b}\right]
\end{aligned}
$$

Finally, the payoff difference between actions $A$ and $B$ is:

$$
\begin{aligned}
G^{A V}(A \mid s)-G^{A V}(B \mid s)= & q(a \mid s)\left[\pi_{A C}^{a} V-\pi_{B C}^{a} v+\left(\pi_{A B}^{a}+\pi_{B A}^{a}\right)(V-v)\right] \\
& -q(b \mid s)\left[\pi_{B C}^{b} V-\pi_{A C}^{b} v+\left(\pi_{A B}^{b}+\pi_{B A}^{b}\right)(V-v)\right] .
\end{aligned}
$$

\subsection{Equilibrium Analysis}

The action set under approval voting is an extension of the action set under plurality. Therefore, in a common value setting as ours, there is always an equilibrium in AV for which the welfare is (weakly) higher than for any equilibrium in plurality (Ahn and Oliveros 2011, proposition 1). ${ }^{28}$ Furthermore, our setup imposes that the size of the minority is large which, as we observed in Section 3, implies that the probability of being pivotal between $A$ and $B$ is always zero under plurality. Theorem 1 directly follows from that fact and from $(15-17)$ :

Theorem 1 There always exists a sincerely stable equilibrium in AV for which expected welfare is strictly higher than for any equilibrium in plurality. In that equilibrium, some voters must double vote, and $\sigma_{s_{A}}(A), \sigma_{s_{B}}(B)>0$.

\footnotetext{
${ }^{28}$ Ahn and Oliveros (2011) exploit McLennan (1998) to show that, in a common value setup as ours, one can rank equilibrium outcomes under approval voting as opposed to some other plurality and negative voting. By revealed preferences, since the action set in the two other rules is a strict subset of the action set under AV, "the maximal equilibrium utility under approval voting is greater than or equal to the maximal equilibrium utility under plurality voting or under negative voting." (p. 3).
} 
Proof. See Appendix A3.

The intuition for this result is as follows: when one compares the set of undominated actions in plurality and in $\mathrm{AV}$, one sees that the only relevant difference is the possibility to double vote $A B$. As emphasized above, double voting $A B$ can be reinterpreted as abstaining between $A$ and $B$ while cumulating the chances of being pivotal against $C$. Now, any strategy profile in plurality imposes that none of the "other voters" plays $A B$. Thus, any voter must realize that she can never be pivotal between $A$ and $B$ with a single vote. In contrast, her expected utility increases strictly by double voting $A B$ rather than mimicking the other voters' strategy profile. ${ }^{29}$ It thus follows that:

Corollary 1 The strategies that are an equilibrium in plurality cannot be an equilibrium in AV. In particular, Duverger's Law equilibria do not exist under AV.

Moreover, since voters have common valued preferences, if such a deviation is beneficial for one voter, it must also increase all the other voters' expected utility. Double voting has pros and cons in terms of the election outcome. On the one hand, it reduces the risk that $C$ wins the election. On the other hand, a voter who double votes does not exploit her signal, which may reduce the probability that $A$ wins in state $a$ and $B$ wins in state $b$. Yet, there cannot be so much double voting that information aggregation would be made impossible:

Corollary 2 Pure double voting is never an equilibrium in $A V$.

The reason is straightforward: if all the other voters double vote, then voter $i$ knows (a) that her vote cannot be pivotal against $C$ and (b) that she is as likely to be pivotal in state $a$ as in state $b$. Hence, her preferred reaction is to vote sincerely and reveal her signal.

Pure double voting has been termed the Burr dilemma by Nagel (2007), who argues that approval voting is inherently biased towards such ties. He documents this with the "[approval] experiment [that] ended disastrously in 1800 with the infamous Electoral College tie between Jefferson and Burr". Lemma 2 shows why such a "disaster" cannot happen in equilibrium of an election which is not dictated by party discipline.

\footnotetext{
${ }^{29}$ To repeat, this is due to the fact that we focus on the case of large minorities. If the size of the minority, $n_{C}$, falls towards zero, then the propensity to double vote may well drop to zero as well (see Bouton and Castanheira, 2012).
} 
Together, Corollaries 1 and 2 show that a voter's best response is to double vote if the other voters single vote "excessively" and to single vote sincerely if the other voters double vote "excessively". In a large Poisson game setup, Bouton and Castanheira (2012) shows that this pattern is monotonic, and that the relative value of the double and single votes cross only once. In other words, approval voting displays a unique equilibrium. Our setup differs in two respects. First, we are not focusing on arbitrarily large electorates as they do. Second, in our setup the size of both the majority and the minority $\left(n\right.$ and $\left.n_{C}\right)$ are fixed. The small-sample and large-sample properties of such voting games can be quite different from one another. For instance, we already emphasized that three-way ties can be neglected in large samples, but not in small samples. This implies that one cannot establish a proof of equilibrium uniqueness for any electorate size and signal distribution. Yet, our next theorem can pinpoint precise voting patterns for any interior equilibrium:

Theorem 2 Whenever both $s_{A^{-}}$and $s_{B}$-voters adopt a nondegenerate mixed strategy, then it must be that voters with signal $s_{A}$ only mix between $A$ and $A B$, and voters with signal $s_{B}$ only mix between $B$ and $A B$.

Proof. See Appendix A3.

This theorem builds on the comparison between the preferences of $s_{A}$ and $s_{B}$ voters: conjecture for instance a case in which the former play $B$ with strictly positive probabilities. Since a voter with signal $s_{B}$ values $B$ even more, it must only play $B$, which contradicts the very nature of an interior equilibrium. To extend this result to equilibria in which (one of the two groups of) voters play degenerate strategies, we would have to focus on larger electorates, which is not the purpose of our analysis. Indeed, Bouton and Castanheira (2012) show that the equilibrium is unique and achieves Full Information and Coordination Equivalence. On the other hand, in small electorate, we need to rely on numerical simulations to study the properties of any such game for a given set of parameters. For all the parametric values we checked, the equilibrium was unique and such that voters with signal $s_{A}$ never play $B$ (i.e. they mix between $A$ and $A B$ ), and voters with signal $s_{B}$ never play $A$. This held both for interior equilibria and for equilibria in which (one of the two sets of) voters play a degenerate strategy.

Two additional examples are useful to better understand the features and comparative statics of voting equilibria in $\mathrm{AV}$ : 
Example 3 Consider the same set of parameters as in example 1: $n=12, n_{C}=7$ or 9, and $r\left(s_{A} \mid a\right)=r\left(s_{B} \mid b\right)=2 / 3$. The two states of nature are equally likely, and the payoffs are: $V=200, v=110$, while the status quo yields a utility of 20.

As emphasized above, there is a unique equilibrium under approval voting for these parameter values (as for all the other parameter values we checked). ${ }^{30}$ It is such that:

$$
\begin{aligned}
& \sigma_{s_{A}}(A)=\sigma_{s_{B}}(B)=0.64 \text { and } \sigma_{s_{A}}(A B)=\sigma_{s_{B}}(A B)=0.36 \text { when } n_{C}=7, \\
& \sigma_{s_{A}}(A)=\sigma_{s_{B}}(B)=0.30 \text { and } \sigma_{s_{A}}(A B)=\sigma_{s_{B}}(A B)=0.70 \text { when } n_{C}=9 .
\end{aligned}
$$

These equilibrium profiles imply that A wins with a probability of $82 \%$ in state a, as does $B$ in state $b$, whereas $C$ 's probability of winning is below $1 \%$ when $n_{C}=7$. When $n_{C}=9$, $A$ wins with a probability of $73 \%$ in state a (as does B in state b), whereas $C$ 's probability of winning remains as low as 1.5\%. These values should be contrasted with the sincere voting equilibrium in plurality (see example 1), in which the probability of selecting the best outcome was substantially lower, whereas the risk that $C$ wins was substantially larger.

Comparing equilibrium behavior with $n_{C}=7$ and $n_{C}=9$ in Example 3 shows that the larger $n_{C}$, the more double voting in equilibrium. This pattern was found to be monotonic and consistent across numerical examples for any value of $n$ and signal structures.

Example 4 Consider the same set of parameters as in Example 3, except for $r\left(s_{A} \mid a\right)=$ 8/9. This reproduces the biased signal setup of Example 2. Like in the previous example, the equilibrium is unique. It yields: $\sigma_{s_{A}}(A)=0.26<\sigma_{s_{B}}(B)=0.52$ and $\sigma_{s_{A}}(A B)=$ $0.74>\sigma_{s_{B}}(A B)=0.48$. This equilibrium profile implies that $A$ wins with a probability of $87 \%$ in state a, whereas $B$ wins with a probability of $90 \%$ in state $b . C$ 's winning probabilities are $0.5 \%$ in state $a$ and $2.8 \%$ in state $b$.

The equilibrium with biased information has the property that the voters with the most abundant signal should single vote less than the voters with the least abundant signal. The rationale for this result might be obvious to the readers knowledgeable about the Condorcet Jury Theorem: if $s_{A^{-}}$and $s_{B}$-voters were to single vote with the same

\footnotetext{
${ }^{30}$ In the strategy space $\left(\sigma_{s_{A}}(A), \sigma_{s_{B}}(B)\right)$, there is a unique cutoff for which $G\left(A \mid s_{A}\right)=G\left(A B \mid s_{A}\right)$, and the same holds for $G\left(B \mid s_{B}\right)=G\left(A B \mid s_{B}\right)$. The equilibrium lies at the intersection between these two reaction functions.
} 
intensity, $A$ 's winning probabilities would be disproportionately higher than $B$ 's. Moreover, the pivot probabilities between $A$ and $B$ would be lower in state $a$ than in state $b$, which should induce all voters to put more value on being pivotal in favour of $B$. An empirical question is whether actual voters would best respond in this way or whether this type of reasoning is too abstract.

\section{$5 \quad$ Experimental Design and Procedures}

\subsection{Experimental Design}

To test our theoretical predictions we ran controlled laboratory experiments. Subjects were introduced to a game that had the very same structure as the one presented in Section 2. All participants were given the role of an active voter, whereas passive voters were simulated by the computer. ${ }^{31}$ Following the experimental literature on the Condorcet Jury Theorem initiated by Guarnaschelli et al. (2000), the two states of the world were called blue jar and red jar, whereas the signals were called blue ball and red ball. The red jar contained six red balls and three blue balls. Depending on the treatment, the blue jar contained either six blue and three red balls (unbiased signals) or eight blue and one red ball (biased signals). One of the jars was selected randomly by the computer, with equal probability. The subjects were not told which jar had been selected, but they received a ball randomly drawn from the selected jar. After seeing their ball, each subject could vote from a set of three candidates: blue, red or gray. ${ }^{32}$ Blue and red were the two majority candidates and gray was the Condorcet loser. Subjects were told that the computer would cast $n_{C}$ votes for gray in each election $\left(n_{C}\right.$ varied across treatments).

The subjects' payoff depended both on the color of the selected jar and that of the winner of the election. If the color of the winner matched the color of the jar, the payoff to all members of the group was 200 euro cents. If the winner was blue and the jar red or the other way around, the payoff to all members was 110 cents. Finally, if gray won, the

\footnotetext{
${ }^{31}$ Morton and Tyran (2012) show that preferences in one group are not affected by preferencs of an opposite group. Therefore, we conjecture that having computerized rather than human subjects did not change the behavior of majority voters in a significant way. Having partisans (who are equivalent to our passive voters) simulated by the computer has been used in previous studies - see Battaglini et al. (2008, 2010).

${ }^{32}$ The colors that we used in the experiments were blue, red and black. Throughout the paper, however, we refer to blue, red and gray respectively.
} 


\begin{tabular}{ccccccc} 
Treatment & $\begin{array}{c}\text { Voting } \\
\text { rule }\end{array}$ & $\begin{array}{c}\text { Minority } \\
\text { size }\left(n_{c}\right)\end{array}$ & $\begin{array}{c}\text { Precision } \\
\text { Blue State }\end{array}$ & $\begin{array}{c}\text { Precision } \\
\text { Red State }\end{array}$ & $\begin{array}{c}\text { Sessions / } \\
\text { Ind. Obs. }\end{array}$ & $\begin{array}{c}\text { Group } \\
\text { numbers }\end{array}$ \\
\hline PL7 & Plurality & 7 & $2 / 3$ & $2 / 3$ & $3 / 6$ & $1-6$ \\
PL9 & Plurality & 9 & $2 / 3$ & $2 / 3$ & $3 / 6$ & $7-12$ \\
AV7 & Approval & 7 & $2 / 3$ & $2 / 3$ & $3 / 6$ & $13-18$ \\
AV9 & Approval & 9 & $2 / 3$ & $2 / 3$ & $3 / 6$ & $19-24$ \\
PL7B & Plurality & 7 & $8 / 9$ & $2 / 3$ & $3 / 6$ & $25-30$ \\
AV7B & Approval & 7 & $8 / 9$ & $2 / 3$ & $3 / 6$ & $31-36$ \\
\hline
\end{tabular}

Table 1: Treatment overview. Note: ind. obs. stands for "individual observations".

payoff to all members was 20 cents.

We consider three treatment variables, which leads to six different treatments. The first variable is the voting mechanism: in $P L$ treatments, the voting mechanism was plurality. In this case, subjects could vote for only one of the three candidates. In $A V$ treatments, the voting mechanism was approval voting. In this case, voters could vote for any non-empty subset of candidates. ${ }^{33}$ With either mechanism, the candidate with the most votes wins, and ties were broken with equal probability. The second variable is the size of the minority, $n_{C}$, which was set to either 7 or 9 . We will refer to them as small and large minority. Finally, the third variable is whether the signal structure is unbiased or biased. In unbiased treatments, signal precision was identical across states and set to $r($ blue ball $\mid$ blue jar $)=$ $r($ red ball $\mid$ red jar $)=\frac{2}{3}$. In biased treatments (which we indicate by $\left.B\right)$, the precision of the signal was the same in state red, whereas $r$ (blue ball | blue jar) was increased to $\frac{8}{9}$. Table 1 provides an overview of the different treatments.

\subsection{Experimental Procedures}

Experiments were conducted at the BonnEconLab of the University of Bonn between July 2011 and January 2012. We ran a total of 18 sessions with 24 subjects each. No subject participated in more than one session. Students were recruited through the online recruitment system ORSEE (Greiner 2004) and the experiment was programmed and conducted with the software z-Tree (Fischbacher 2007).

All experimental sessions were organized along the same procedure: subjects received

\footnotetext{
${ }^{33}$ As in Guarnaschelli et al. (2000), abstention was not allowed (remember that abstention is always a strictly dominated action). In a similar setting, Forsythe et al (1993) allowed for abstention and found that the abstention rate was as low as $0.65 \%$.
} 
detailed written instructions, which an instructor read aloud (see Appendix B). Each session proceeded in two parts: in the first part, subjects played in fixed groups one of the treatments described for 100 periods. ${ }^{34}$ Before starting, students were asked to answer a questionnaire to check their full understanding of the experimental design. In the second part, subjects received new instructions, and then made 10 choices in simple lotteries, as in Holt and Laury (2002). We ran this second part to elicit subjects' risk preferences.

To determine payment at the end of the experiment, the computer randomly selected four periods from the first part and one lottery from the second part. ${ }^{35}$ In total, subjects earned an average of $€ 13.47$, including a showup-fee of $€ 3$. Each experimental session lasted approximately one hour.

\section{Experimental Results}

We present our experimental results in four distinct sections. Section 6.1 presents the outcomes when information is unbiased, and Section 6.2 when it is biased. Section 6.3 turns to individual behavior and extends the model to asymmetric equilibria. Finally, section 6.4 summarizes the main findings regarding outcomes and welfare.

\subsection{Unbiased Treatments}

\subsubsection{Plurality}

As shown in section 3, two types of equilibria coexist under plurality when information is unbiased: Duverger's Law and sincere voting equilibria. In the former type of equilibria, participants should disregard their signal and coordinate on voting always blue or always red. In the latter instead, participants should vote their signal. Table 2 shows the average frequencies with which subjects voted sincerely (we call this voting the signal), for the majoritarian party opposite to the signal (we will call this voting opposite) or for gray. ${ }^{36}$

\footnotetext{
${ }^{34}$ In the setup of the Condorcet Jury Theorem, Ali et al (2008) find no significant difference between random matching (which they call ad hoc committees) and fixed matching (which they call standing committees).

${ }^{35}$ In the first round of experiments (the seven sessions with the groups 1, 2, 7, 8, 9, 10, 13, 14, 15, 16, 19, 20, 21 and 22), we selected seven periods to determine payment. We reduced this to four periods after realizing that the experiment had taken much less time than expected. We find no difference in behavior between these two sets of sessions.

${ }^{36}$ The figures identified with a ${ }^{* *}$, report the predicted voting pattern for the last 50 periods, conditional on the color (blue or red) on which the group coordinated. For instance, if the group coordinated on blue,
} 


\begin{tabular}{ccccccc} 
& Minority & & Periods & Periods & \multicolumn{2}{c}{ Equilibrium } \\
\cline { 5 - 6 } Treatment & Size & & $1-50$ & $51-100$ & Sincere Voting & Duverger's Law \\
\hline PL7 & Small & Signal & 91.80 & 90.94 & 100.00 & 50.00 \\
& & Opposite & 7.78 & 8.89 & - & 50.00 \\
& & Gray & 0.42 & 0.17 & - & - \\
\hline PL9 & Large & Signal & 68.47 & 59.25 & 100.00 & $48.92^{*}$ \\
& & Opposite & 31.11 & 40.67 & - & $51.08^{*}$ \\
& & Gray & 0.41 & 0.08 & - & - \\
\hline
\end{tabular}

Table 2: Aggregate voting behavior in plurality treatments with unbiased information, separated by first and second half, and equilibrium predictions. * In the case of Duverger's Law in PL9, the prediction is adjusted to the color that each group converged to.

In the presence of a small minority, the participants' voting behavior is consistent with the sincere voting equilibrium: taking an average across all groups and periods, $91.38 \%$ of the ballots were sincere in PL7. This finding holds across groups: there were at least $86.42 \%$ of sincere ballots in each independent group. As shown in Table 3, this behavior is quite stable over time: we regress the frequency of "voting the signal" on the period number, and find that the coefficient is not significantly different from zero. Most deviations from sincere voting behavior consisted of "voting opposite". Part of this might be related to subjects misunderstanding the fact that random events are independent across periods. Such misunderstanding has been observed frequently in the literature. ${ }^{37}$ Finally, less than $0.5 \%$ of the observations were votes for gray.

Voting behavior is substantially different in the presence of a large minority (PL9). First, only $63.86 \%$ of the observations are consistent with sincere voting behavior. Second, there is a clear temporal trend: as shown in Table 3, the frequency of voting the signal significantly decreases over time: the predicted frequency of sincere voting drops from $73.90 \%$ in the first periods to $53.70 \%$ in the last period. That is, participants begin the experiment by voting sincerely (94.44\% of them voted their signal in the first period), and then they adjust their behavior by increasingly voting against their signal. This pattern is

\footnotetext{
and if $40 \%$ of the voters obtain a blue ball in a given draw, then $40 \%$ should play "signal" and $60 \%$ play "opposite".

${ }^{37}$ The misperception of the independence between independent events is known in the lituerature as the gambler's fallacy. More precisely, the gambler's fallacy is the mistaken notion that the likelihood of an event that occurs with a fixed probability increases or decreases depending upon recent occurrences. The gambler's fallacy has been documented extensively in laboratory experiments, survey data and field experiments (see Tversky and Kahneman 1971 or Clotfelder and Cook 1993). In our experiment, the gambler's fallacy might lead subjects to disregard signals on the ground that the perceived likelihood of the signal being wrong is higher than the likelihood of the signal being right after some particular histories.
} 


\begin{tabular}{lcc} 
& PL7 & PL9 \\
\hline \hline Period & -0.0000983 & $-0.00204^{* * *}$ \\
& $(0.000202)$ & $(0.000182)$ \\
Constant & $0.919^{* * *}$ & $0.741^{* * *}$ \\
& $(0.0150)$ & $(0.0248)$ \\
\hline Observations & 7200 & 7200 \\
$\mathrm{R}^{2}$ & 0.0001 & 0.0150 \\
\hline \hline
\end{tabular}

Table 3: Linear Regression of the frequency of voting the signal on the period number and a constant, clustering by independent group, for each treatment with plurality and unbiased information. Standard errors in parentheses. ${ }^{*} p<0.10,{ }^{* *} p<0.05,{ }^{* * *} p<0.01$

fully consistent with the progressive shift from a sincere voting equilibrium to a Duverger's Law equilibrium. Figure 1 illustrates this shift by plotting the observed frequency of voting blue, red and gray (irrespective of the signals subjects receive) for each group in the PL9 treatment. The horizontal dashed line displays the minimal vote share required to defeat gray (in case nobody plays the dominated strategy of voting gray). As one can see, all six groups did converge to a Duverger's Law equilibrium: groups 7 and 9 converged to voting blue regardless of one's signal, whereas the other groups converged to voting red.

Two additional elements emerge from these data on PL9. First, which Duverger's Law equilibrium they converge to is actually decided very early: all but one group ended up coordinating on the first color obtaining strictly more than six of the majority votes (it happened in period 1 for four groups and in period 2 for one group). ${ }^{38}$ This is in line with the findings of Forsythe et al (1993, p235): "a majority candidate who was ahead of the other in early elections tended to win the later elections, while the other majority candidate was driven out of subsequent races". Second, even though this pattern is obvious with hindsight, the groups' shifting from sincere voting towards a Duverger's Law equilibrium was fairly slow. This is exemplified by the minimum period from which either blue or red repeatedly obtained enough votes to win: the period number was respectively 50,59, 83, 63, 21 and 26 for groups 7-12.

This raises two interlinked questions: (1) starting at the same sincere voting equilibrium as "small minority" groups, why do all "large minority" groups switch to a Duverger's Law equilibrium? (2) why is that switch slow? Answering the first question is relatively

\footnotetext{
${ }^{38}$ The exception is group 11 in which blue got seven votes in the first period and then red received more votes from period 2 onwards.
} 

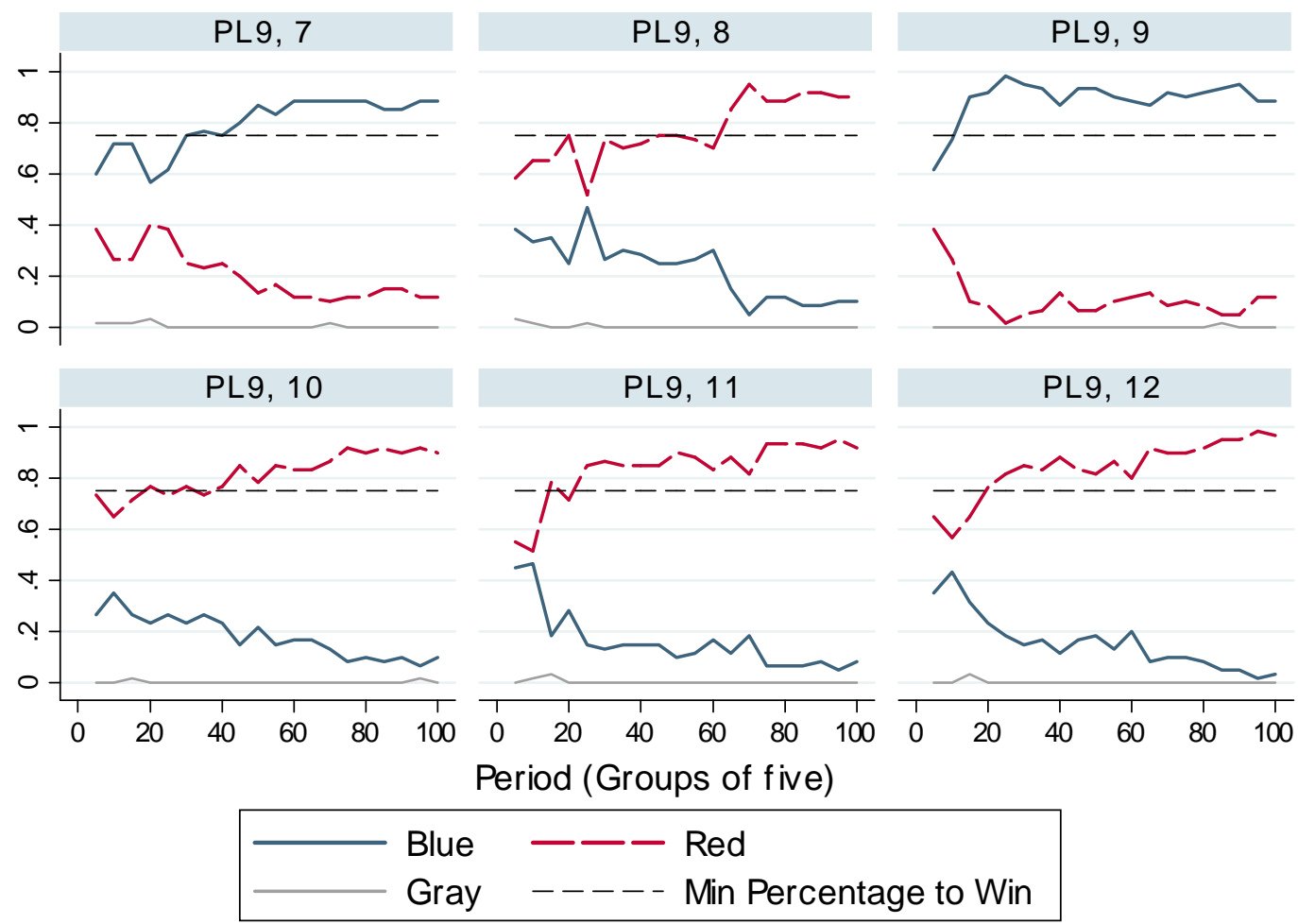

Figure 1: Frequence of voting blue, red and gray irrespective of the signal in groups of treatment PL9U. The dashed line indicates the minimum frequence of vote share required to defreat gray (in case nobody from the majority votes for the condorcet loser). 
straightforward: facing a large minority means that voters face an expected utility of 69.76 in PL9, instead of 152.76 in PL7. This should be compared to an expected utility of 155 in a Duverger's Law equilibrium. The incentive to get away from the sincere voting equilibrium is thus substantially higher in PL9.

Addressing the second question is more difficult. Going back to theory, we can compute a voter's best response for any strategy profile by the other 11 voters. The phase diagram in Figure 2 illustrates this graphically. The horizontal axis displays the other blue voters' propensity to vote blue, and the vertical axis displays the other red voters' propensity to vote red. The solid curve represents the locus of the others' strategies for which a voter is indifferent between playing blue or red if she receives a blue ball. To the left of that curve, the payoff of red is higher than that of blue, and conversely to the right of the curve. The dashed curve represents the equivalent locus for a voter who receives a red ball. Above the curve, she prefers the payoff of playing red is higher than that of blue, and conversely below the curve. The arrows in Figure 2 display the attraction zones of each of the three equilibria mentioned: sincere voting in the top right corner, and the two Duverger's Law equilibria in the bottom right and top left corners. As it appears clearly, the attraction zone of the sincere voting equilibrium is much larger in PL7 than in PL9, but it is still pretty large even in PL9. Within this attraction zone, after receiving her signal, a voter thus faces two opposite incentives: a static incentive to reveal her signal, and a dynamic incentive to coordinate towards one of the two Duverger's Law equilibria. This helps explain why convergence is slow, despite the dynamic incentive. Moreover, the larger dynamic incentive and the smaller attraction zone in PL9 help explain why all groups ended up moving away from sincere voting.

\subsubsection{Approval Voting}

Table 4 summarizes behavior in AV treatments. It displays the frequencies with which subjects single vote for their signal, double vote for the two majority candidates, single vote opposite to their signal, and vote for gray (possibly in combination with another candidate).

These two treatments reproduce the parametric cases covered in Example 3, which we found to display a unique symmetric equilibrium. In that equilibrium a voter should only 

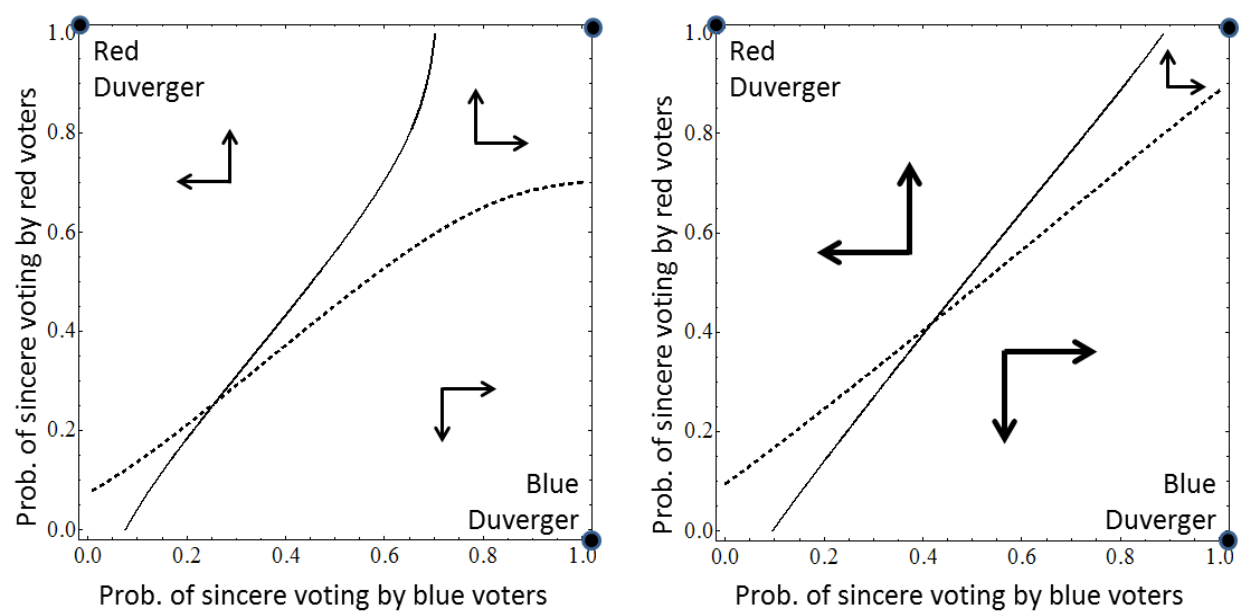

Figure 2: Phase diagram of treatments PL7 and PL9. The horizontal axis displays the probability of sincere voting by blue voters while the vertical axis displays the probability of sincere voting by red voters. The solid line indicates the indifference curve for the blue voters, while the dashed line indicates the indifference curve for the red voters.

\begin{tabular}{cclccc} 
& & & Periods & Periods & \\
Treatment & Minority Size & & $1-50$ & $51-100$ & Equilibrium \\
\hline AV7 & Small & Signal & 70.92 & 71.94 & 64.00 \\
& & Double Vote & 22.22 & 24.36 & 36.00 \\
& & Opposite & 6.50 & 3.69 & - \\
& & Gray & 0.36 & 0.00 & - \\
\hline AV9 & Large & Signal & 47.08 & 43.33 & 30.00 \\
& & Double Vote & 45.67 & 51.64 & 70.00 \\
& & Opposite & 6.86 & 4.97 & - \\
& & Gray & 0.39 & 0.06 & - \\
\hline
\end{tabular}

Table 4: Aggregate voting behavior in approval voting treatments with unbiased information. Gray refers to voting for gray or a combination of gray and others. 
single vote for her signal or double vote Blue and Red. A huge majority of actions were in line with this theoretical prediction: $94.73 \%$ in the case of AV7 and $93.86 \%$ in the case of AV9. One could think that AV involves higher complexity and therefore higher frequency of mistakes, but we actually observed the opposite. Define mistakes as playing an action that is not a best response to the equilibrium. That is, in PL7, AV7 or AV9, a subject made a mistake when she voted opposite to her signal or for gray; in PL9, she made a mistake when voting for another color than the one the rest of the group converged to. We find that subjects made more mistakes under plurality than under approval voting. In treatments with a small minority, the percentages of mistakes in the second half (where equilibrium selection is clearer) were $3.69 \%$ in AV7 as opposed to $9.06 \%$ in PL7. In treatments with a large minority, they represented $5.03 \%$ of the ballots in AV9 as opposed to $11.06 \%$ in PL9. These differences are significant in both cases (Mann-Whitney, $\mathrm{z}=2.082, \mathrm{p}=0.0374$ with $c=7$ and $\mathrm{z}=1.925, \mathrm{p}=0.0542$ with $c=9) .{ }^{39}$

The second theoretical prediction drawn from Example 3 refers to the effect of minority size: there should be an increase in the frequency of double voting. The rationale is that voters need to double vote more to contain the risks of election of the minority candidate, gray. Table 4 shows that this is indeed the way in which the subjects adapted their voting behavior: the percentage of double voting was multiplied by more than two, from $23.29 \%$ in treatment AV7 to $48.66 \%$ in treatment AV9. This difference is significant at 1\% (Mann-Whitney, $\mathrm{z}=2.722, \mathrm{p}<0.01$ ).

Although the comparative statics go in the direction predicted by theory, one should notice that there is a considerable difference between the data and the theoretical predictions: the amount of double-voting was well below the prediction. In treatment AV7, the observed amount of double-voting was 24.36 instead of 36.00 in theory. In treatment AV9 it was 51.64 instead of 70.00 . These differences are significant at $5 \%$ in both cases (Mann-Whitney, $\mathrm{z}=2.201, \mathrm{p}<0.05$ ). Section 6.3 returns to this discrepancy to show that asymmetric equilibria help explain this gap.

\footnotetext{
${ }^{39}$ One could also define mistakes as voting for gray (or gray with something else). In this case as well, plurality leads to more mistakes than $\mathrm{AV}$, but these differences are not significant (Mann-Whitney, $\mathrm{z}=$ $1.000, \mathrm{p}=0.3173$ with $c=7$ and $\mathrm{z}=0.561, \mathrm{p}=0.5751$ with $c=9)$.
} 


\subsection{The Effects of Biased Information}

In PL7, we observed that all independent groups coordinated on the sincere voting equilibrium. One reason might be the symmetry between the blue and red signals, which made coordination challenging for the subjects. In treatment PL7B, we instead made the signal structure strongly biased in favor of the blue signal by setting $r$ (blue ball $\mid$ blue jar $)=8 / 9$. So, if the voters were to keep playing sincere, blue would win disproportionately more often than red. Together with Example 2, Propositions 1 and 3 show that voters may still coordinate on either of two potential equilibria. First, they may coordinate on the Duverger's Law outcome. We may even expect that coordination was made simpler: the higher expected vote share of blue may have fostered coordination by red voters towards voting blue. The second possible outcome is the informative equilibrium. Example 2 showed that, to aggregate information, blue voters should then adopt a strictly mixed strategy and vote red with strictly positive probability ( $8.43 \%$ according to Example 2$)$.

In the data, we observe that one independent group (group 28) out of six coordinated on the "blue" Duverger's Law equilibrium, whereas the other five adopted a strategy coherent with the informative equilibrium. Let us analyze each in turn: in group 28, almost all voters casted a blue ballot as of period 31: from that period onwards, blue obtained at least seven votes in all cases, and strictly more than seven in $94.29 \%$ of the cases. ${ }^{40}$ Table 5 below summarizes the behavior of the other 5 independent groups. Focusing on the last fifty periods to account for convergence towards an equilibrium, we observe that blue voters indeed voted red with a probability close to the theoretical prediction, whereas red voters mainly voted sincerely. The difference between these two behaviors is statistically significant (Mann-Whitney, $z=2.023, p<0.043$ ). Moreover, we do not find any significant difference between the theoretical prediction and the observed frequency of voting blue when getting a blue ball (Mann-Whitney, $z=0.405, p=0.6858$ ).

The transition from sincere voting in the first periods towards either of the two types of equilibria illustrates the difficulty of coordinating on one equilibrium when several are available, each with its pros and cons. The main advantage of Duverger's Law equilibria is

\footnotetext{
${ }^{40}$ The fact that the only group that converged to a Duverger's Law equilibrium did coordinate on blue is coherent with the idea that biased signals foster coordination. However, this intuition cannot be tested since there is only one such group to study. Moreover, the results in PL9 offer an alternative rationale, which is that voters coordinate on the color that won in the first period(s). The investigation of which factors dominate equilibrium selection seem an interesting topic for future research.
} 


\begin{tabular}{lccc} 
& Periods & Periods & \\
& $1-50$ & $51-100$ & Equilibrium \\
\hline Signal if blue & 92.99 & 90.75 & 91.53 \\
Opposite if blue & 6.89 & 8.38 & 8.47 \\
Signal if red & 96.39 & 97.48 & 100 \\
Opposite if red & 3.13 & 1.74 & 0 \\
Gray & 0.27 & 0.83 & 0 \\
\hline
\end{tabular}

Table 5: Aggregate voting behavior in treatment PL7B. Group 28 was excluded given that it converged to a Duverger's Law equilibrium.

that it removes uncertainty about the outcome of the election, and ensures that gray can never win. Its main drawback is that there is no aggregation of information. Conversely, the informative equilibrium maximizes the probability of electing the best candidate in each state but does not offer the "safety" of Duverger's Law equilibria: all candidates have a strictly positive probability of winning.

The model helps identify two reasons why the latter equilibrium is more likely to be selected. First, it yields a higher expected payoff than Duverger's Law equilibria (178.37 instead of 155). Second, as identified by the phase diagram displayed in Figure 3, when starting from sincere voting (the top-right corner), the local dynamics of individual best responses point towards the informative mixed strategy equilibrium (the point MSE on the graph) rather than towards either Duverger's Law equilibria.

These data also provide ample evidence to confirm our theoretical prediction that threecandidate equilibria are a natural focal point when voters have common values. It is also noteworthy that voters do not simply play "sincere". In the five groups that selected the informative equilibrium, blue voters actually adjusted their behavior to better aggregate information. While Bhattacharya et al (2012) already confirm that such voting behavior is empirically robust in a two-candidate setting, we believe that our experimental results are the first ones to identify this pattern in a three-candidate setting.

Approval voting offers the opportunity to double vote to achieve the twin objectives of information aggregation and coordination. Treatment AV7B is the same as PL7B with the only difference that subjects can exploit this opportunity. Thus, in this treatment, voters face the more complex challenge of having to deal with a broader choice set but, as identified in Example 4, their task is simplified by the fact that the equilibrium is unique in this setup. Like in the informative equilibrium of PL7B, blue voters should play blue 


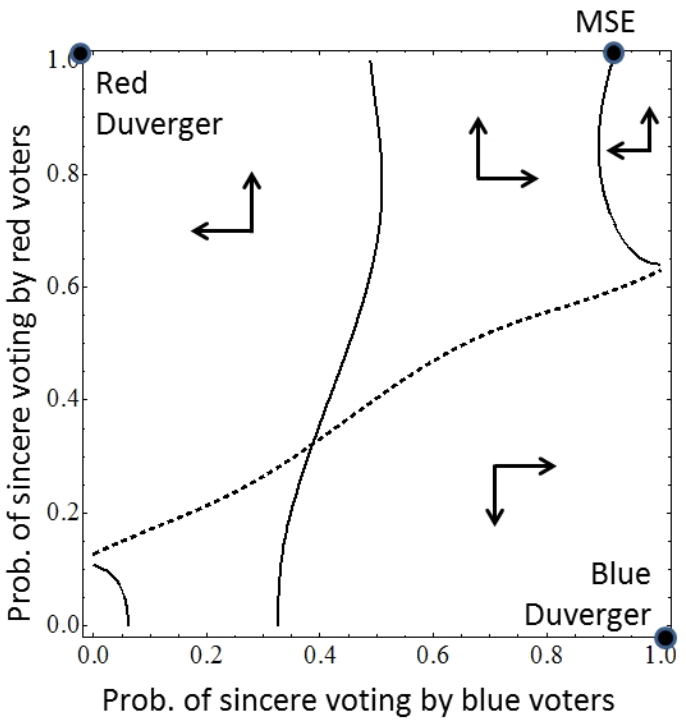

Figure 3: Phase diagram of treatment PL7B. The horizontal axis displays the probability of sincere voting by blue voters, while the vertical axis displays the probability of sincere voting by red voters. The solid line indicates the indifference curve for the blue voters; the dashed line indicates the indifference curve for the red voters.

\begin{tabular}{lccc} 
& Periods & Periods & \\
& $1-50$ & $51-100$ & Equilibrium \\
\hline Signal if blue & 66.95 & 61.16 & 50.1 \\
Double Vote if blue & 29.87 & 37.16 & 49.9 \\
Signal if red & 74.56 & 80.52 & 92.6 \\
Double Vote if red & 20.52 & 17.98 & 7.4 \\
Opposite & 2.94 & 1.56 & 0 \\
Gray & 0.89 & 0.06 & 0 \\
\hline
\end{tabular}

Table 6: Aggregate Voting Behavior in treatment AV7B.

less often than red voters should play red. The difference is that blue voters should double vote instead of voting red. Table 6 confirms that the voters' behavior is in line with this prediction. Actually, the results are even stronger since the difference between the blue and red voters is significant not only for the second half of the sample but for the whole experiment (Mann-Whitney, $\mathrm{z}=2.201, \mathrm{p}=0.028$ ).

\subsection{Individual Behavior}

We begin by describing individual behavior in plurality treatments with unbiased information. These cases do not allow for much variation among players: in treatment PL7 most 


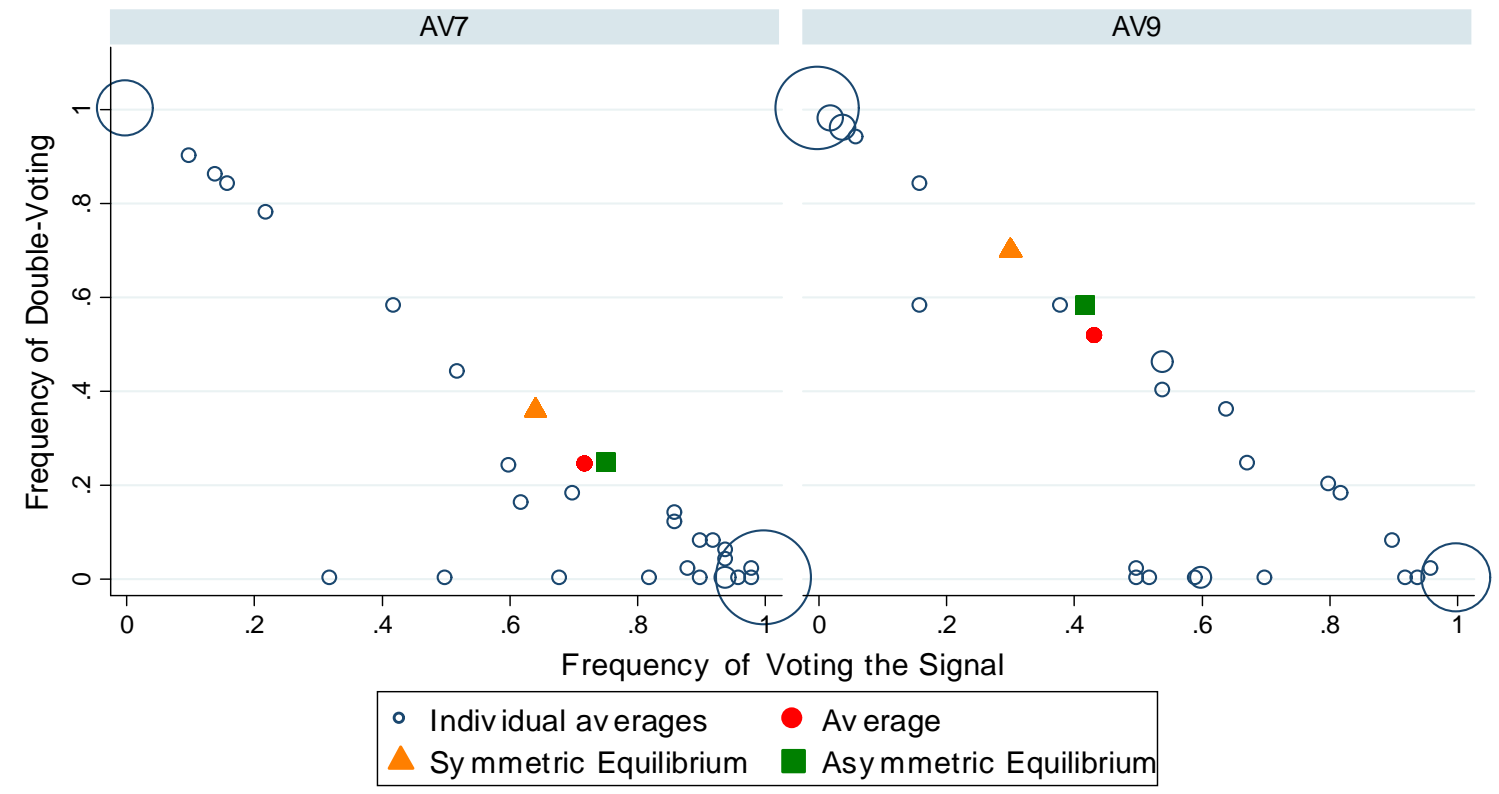

Figure 4: Individual behavior in AV treatments with unbiased information. Each hollow circle in the graph corresponds to the observed frequence of play for one or more subjects. Its size represents the number of subjects who actually adopted that frequence of play. The red circle represents the average frequency of play observed, the orange triangle represents the symmetric equilibrium prediction and the green square represents the asymmetric equilibrium prediction.

subjects voted sincerely throughout the entire experiment: $43.06 \%$ of the participants always did and $88.89 \%$ of the subjects voted sincerely in more than $75 \%$ of the occasions. As we saw, behavior is somewhat different in PL9, since voters always converged to a Duverger's law equilibrium, although slowly. In the last half of the experiment, $88.94 \%$ voted for the color their group converged to.

The case of approval voting is somehow more interesting. Figure 4 disaggregates behavior at the individual level in the last fifty periods of treatments AV7 (left panel) and AV9 (right panel). The horizontal axis plots the frequency of voting the signal and the vertical axis plots the frequency of double voting. Each circle in the graph corresponds to the observed frequency of play for one or more subjects. Its size represents the number of subjects who actually adopted that frequency of play: the bigger the circle, the higher the number of subjects.

According to Theorem 2, subjects should only mix between voting the signal or double- 
voting. If all subjects voted in this way, all the circles should be on the diagonal between $(0,1)$ and $(1,0)$. We observe that most circles are indeed on this diagonal but, instead of observing a large number of voters playing the predicted mixed strategy, we observe significant heterogeneity with two opposite clusters: one that plays the pure strategy of always double voting and another one with subjects who always single vote their signal. The treatment effect observed in Section 6.1.2 is mainly driven by a switch in the relative number of subjects in each cluster.

This pattern pointed at the need to consider asymmetric strategies. Pushing the line of reasoning of Ahn and Oliveros (2011) further, allowing for asymmetric strategies can be interpreted as an extension of the group's choice set, which may only increase expected welfare. Allowing some voters to specialize in double or single voting may produce significant advantages. The challenge is to identify potential equilibria when allowing for asymmetric strategies. We deal with this issue in the next subsection.

\subsubsection{Asymmetric Equilibria with Approval Voting}

In this subsection, we relax the assumption of symmetric strategies, ubiquitous as it is in the voting literature. ${ }^{41}$ That is, we do not force voters who receive the same signal to play the same strategy. The following proposition proves, for a broad set of parameter values (including the ones used in the experiment), the existence of at least one asymmetric equilibrium, i.e. in which voters play asymmetric strategies. We also characterize this asymmetric equilibrium: voters specialize independently of their signal in either single voting or double voting. That is, some voters always single vote and others always double vote. The "single voters" condition their behavior on their signal. If the signal structure is sufficiently unbiased, they all vote sincerely, i.e. $A$ if signal $s_{A}$ and $B$ if signal $s_{B}$. If the signal structure is not sufficiently unbiased, then the voters receiving the less abundant signal (in expectation) vote sincerely whereas those who receive the more abundant signal mix between $A$ and $B$ in order to compensate for the bias. As discussed below, it appears that this equilibrium helps rationalize the behavior observed in the laboratory.

Proposition 4 Suppose that $q(a)=q(b), r\left(s_{A} \mid a\right) \geq r\left(s_{B} \mid b\right)$ and $V \leq 2 v$. Any strategy profile satisfying the following conditions is an equilibrium:

\footnotetext{
${ }^{41}$ There are noticeable exceptions in the literature on the Condorcet Jury Theorem. McLennan (1998), Ladha, Miller and Oppenheimer (2000) or Dekel and Piccione (2000) consider asymmetric equilibria.
} 
1. $2 n_{C}-n+1$ voters always double vote;

2. The rest of the voters single vote informatively with the following probabilities:

$$
\begin{aligned}
& \sigma_{s_{A}}^{1 v}(A)=\left\{\begin{array}{c}
\frac{\left(\frac{r\left(s_{A} \mid a\right)}{r\left(s_{A} \mid b\right)}\right)^{\frac{n-n_{C}}{n-n_{C}-1}}-1}{\left(\frac{r\left(s_{A} \mid a\right)}{r\left(s_{A} \mid b\right)}\right)^{\frac{n-n_{C}}{n-n_{C}-1}} r\left(s_{A} \mid a\right)-r\left(s_{A} \mid b\right)} \text { if }\left(\frac{r\left(s_{A} \mid a\right)}{r\left(s_{A} \mid b\right)}\right)^{\frac{n-n_{C}}{n-n_{C}-1}}>\frac{r\left(s_{B} \mid b\right)}{r\left(s_{B} \mid a\right)} \\
1 \text { if }\left(\frac{r\left(s_{A} \mid a\right)}{r\left(s_{A} \mid b\right)}\right)^{\frac{n-n_{C}}{n-n_{C}-1}} \leq \frac{r\left(s_{B} \mid b\right)}{r\left(s_{B} \mid a\right)}
\end{array}\right. \\
& \sigma_{s_{B}}^{1 v}(B)=1,
\end{aligned}
$$

where $\sigma_{s}^{1 v}(\psi)$ is the probability that a single voter of type s plays action $\psi$.

Proof. See Online Appendix.

Such an asymmetric behavior can be sustained in equilibrium because voters who specialize in single voting perceive the expected effect of any given ballot on the final outcome differently than voters specializing in double voting. In particular, "single voters" are pivotal only when $A, B$, and $C$ receive exactly the same number of votes, whereas "double voters" are pivotal if either $A$ is trailing behind by one vote or if it is leading by one vote. The best responses of these two groups of voters are thus different.

We illustrate Proposition 4 as well as some properties of the asymmetric equilibrium through a numerical example:

Example 5 Assume again (as in example 3, p26): $n=12, n_{C}=7$, and $q\left(s_{A} \mid a\right)=$ $q\left(s_{B} \mid b\right)=2 / 3$. In the asymmetric equilibrium, $2 n_{C}-n+1=3$ voters double vote, and the other 9 single vote their signal.

Compared with the symmetric equilibrium, the aggregate level of double voting decreases from $36 \%$ to $25 \%$, but this is enough to ensure that the Condorcet loser cannot win the election. Indeed, with three double votes, and nine single votes, one of the two majority alternatives must receive at least eight votes, i.e. strictly more than the Condorcet loser. Finally, the likelihood of choosing the best candidate increases from $82 \%$ in the symmetric equilibrium to $85.5 \%$. The better aggregation of information holds even if we abstract from the cases in which the Condorcet loser wins in the symmetric equilibrium. This is because the (expected) number of voters who reveal their information, i.e. the single voters, is larger in the asymmetric equilibrium than in the symmetric one (9 vs. 7.68 ). 


\begin{tabular}{|c|c|c|c|c|c|c|}
\hline Treatment & Minority Size & & $\begin{array}{c}\text { Periods } \\
1-50\end{array}$ & $\begin{array}{c}\text { Periods } \\
51-100\end{array}$ & $\begin{array}{l}\text { Symmetric } \\
\text { Equilibrium }\end{array}$ & $\begin{array}{l}\text { Asymmetric } \\
\text { Equilibrium }\end{array}$ \\
\hline \multirow[t]{4}{*}{ AV7 } & Small & Signal & 70.92 & 71.94 & 64.00 & 75.00 \\
\hline & & Double Vote & 22.22 & 24.36 & 36.00 & 25.00 \\
\hline & & Opposite & 6.50 & 3.69 & - & \\
\hline & & Gray & 0.36 & 0.00 & - & \\
\hline \multirow[t]{4}{*}{ AV9 } & Large & Signal & 47.08 & 43.33 & 30.00 & 41.66 \\
\hline & & Double Vote & 45.67 & 51.64 & 70.00 & 58.33 \\
\hline & & Opposite & 6.86 & 4.97 & - & \\
\hline & & Gray & 0.39 & 0.06 & - & \\
\hline
\end{tabular}

Table 7: Aggregate voting behavior in AV treatments with unbiased information (Revisited). Gray refers to voting gray or a combination of gray and others.

As shown in Table 7, such asymmetric equilibria appear to organize laboratory data better than the symmetric equilibrium. In treatment AV7, the predicted level of double voting in the asymmetric equilibrium is $25 \%$ compared to the observed $24.46 \%$. The difference is not significant (Wilcoxon, $\mathrm{z}=-0.524 \mathrm{p}=0.6002$ ). In the case of AV9, the predicted level of double voting is $58.33 \%$ compared to the observed $51.64 \%$. The difference is significant (Wilcoxon, $\mathrm{z}=2.201, \mathrm{p}=0.0277$ ).

The equilibrium described in Proposition 4 also makes an interesting prediction for the biased treatment AV7B. In the asymmetric equilibrium, the level of double voting is independent of the signal structure. This is not what we observe in the data (see Table 6). As shown in Section 6.2, we observe significantly different behavior depending on the signal received. However, as with treatment AV7, there seem to be significant differences in behavior between subjects. Although it is beyond the scope of this paper, it might be useful to explore different types of asymmetric equilibria in this type of setting.

\subsection{Welfare and Outcomes}

Previous multicandidate election setups used in laboratory experiments were based on theories which were inconclusive when it came to comparing welfare across voting mechanisms. The theoretical predictions of Myerson and Weber (1993) used in Forsythe et al. (1996), for instance, do not make a clear-cut prediction when comparing plurality and approval voting. ${ }^{42}$ A valuable feature of our common value setup is that it allows us to

\footnotetext{
${ }^{42}$ Forsythe et al. (1993) does make the prediction that approval voting performs weakly better than the Borda Rule. In one of the equilibria, approval voting can replicate the Borda rule. In the other two equilibria, AV dominates Borda. In contrast, they cannot make clear predictions concerning plurality
} 


\begin{tabular}{ccccc} 
& Periods & Periods & \multicolumn{2}{c}{ Equilibrium } \\
\cline { 4 - 5 } Treatment & $1-50$ & $51-100$ & Symmetric* $^{*}$ & Asymmetric \\
\hline PL7 & 136.70 & 138.50 & 154.87 & - \\
PL9 & 101.15 & 147.80 & 156.20 & - \\
PL7B & 169.85 & 171.95 & 178.76 & - \\
\hline AV7 & 167.00 & 183.95 & 179.65 & 189.80 \\
AV9 & 146.75 & 168.95 & 164.70 & 181.10 \\
AV7B & 188.90 & 192.50 & 193.58 & 194.68 \\
\hline
\end{tabular}

Table 8: Average payoff and theoretical predictions. * In the case of Plurality, equilibrium predictions refer to the equilibrium where experimental groups converged to.

make clear predictions in terms of welfare: in equilibrium, the active voters' payoff should be strictly higher with approval voting than in plurality (Theorem 1, p.23).

Table 8 , columns 2 and 3, displays the average payment obtained by the subjects in each treatment, respectively for the first and second fifty periods. Comparing PV and AV treatments two by two, one can observe that realized payoffs are systematically higher in AV treatments than in the associated PV treatment. All these differences are significant at a $1 \%$ confidence level. ${ }^{43}$

It is also interesting to see the effect of the size of the minority. The comparative statics are not clear in the case of plurality, given equilibrium multiplicity. The expected payoff should be strictly decreasing in $n_{C}$ in an informative equilibrium, whereas it is independent of $n_{C}$ in a Duverger's Law equilibrium. In the case of our experiment, the expected payoff of Duverger's Law equilibria is 155 . Table 8 shows an interesting reversal when comparing PL7 and PL9: in the first half, the average payoff is higher in treatment PL7 than in treatment PL9, while the opposite is true for the second half. This is explained by the progressive switch towards a Duverger's Law equilibrium in all independent groups under PL9, and the selection of the sincere voting equilibrium by all groups in PL7. The latter happens in spite of the fact that Duverger's Law equilibria payoff dominate sincere voting in both treatments. On the other hand, the slow convergence process in the first half of PL9 treatments explains why payoffs are higher in PL7 than in PL9 treatments for the first 50 periods. Across the entire experiment session, payoff is lower under PL9 than under PL7 (Mann Whitney test, $\mathrm{z}=1.922, \mathrm{p}$-value $=0.0547$ ).

\footnotetext{
versus $\mathrm{AV}$.

${ }^{43}$ Mann-Whitney tests are: $\mathrm{z}=2.882$ and $\mathrm{p}$-value 0.0039 for $\mathrm{AV7}-\mathrm{PV} 7, \mathrm{z}=2.722$ and $\mathrm{p}$-value $=0.0065$ for AV9-PV9, and $\mathrm{z}=2.913$ and $\mathrm{p}$-value $=0.0036$ for AV7B-PV7B.
} 
In the case of approval voting the theoretical prediction is unambiguous since there is a unique equilibrium. Both in the symmetric and asymmetric equilibria, the voters' payoffs is predicted to be strictly decreasing with the size of the minority. As one can see from Table 8, this is what we observe in the data. These differences are statistically significant (Mann Whitney test, $\mathrm{z}=2.242, \mathrm{p}$-value $=0.0250$ ). Looking more closely at actual outcomes (see Appendix A3), one can check that this is due to a remarkable increase in the frequence of victory of the best candidate, combined with a drop in the frequence of victory of the Condorcet loser. This observation can be made in all two-by-two comparisons, including PL9 against AV9, because Gray won $10 \%$ of the times even in the second half of the PL9 experiment, due to slow convergence towards the equilibrium.

\section{Conclusions}

In this paper we studied the properties of plurality and approval voting both theoretically and experimentally. We considered a case in which the majority is divided between two alternatives as a result of information imperfections and the minority backs a third alternative, which the majority views as strictly inferior. The majority thus faced two problems: aggregating information and coordinating to defeat the minority candidate.

In plurality, we identify two distinct types of equilibria. First, in Duverger's Law equilibria, all majority voters coordinate their ballots on a same majority alternative. This means that they can fully insure against a victory of the minority alternative, but they have to give up any possibility of aggregating information. Second, there also exists an informative equilibrium, in which majority voters split their ballots between the two majority alternatives. In equilibrium, the majority alternative with the largest expected voter share is the full information Condorcet winner. The drwaback of this this strategy is that the Condorcet loser may win the election. Interestingly, this equilibrium is not "knife edge": strictly more than two candidates receive different but substantial vote shares. This theoretical finding helps rationalize some empirical regularities in the literature that are oft-considered as supporting evidence for the lack of a "rational-instrumental" voting behavior.

In approval voting, the structure of incentives is quite different: the threat posed by the minority candidate provides an incentive to double vote in order to reduce the relative 
size of the minority. However, this does not produce indeterminate results: there is no equilibrium in which all majority voters double vote. Hence, voters in the majority keep aggregating information. We actually find that, in comparison with any potential equilibrium in plurality, there exists an equilibrium in AV that produces strictly higher expected welfare. Next, we characterize the equilibrium with approval voting in any interior equilibrium: each voter must adopt a mixed strategy of either single voting for her preferred candidate only, or double voting for both majority alternatives.

We then tested our predictions with laboratory experiments. They revealed very interesting patterns and supported most of our theoretical predictions. We first studied setups in which information is symmetric across states: under plurality, we observed the emergence of both informative and Duverger's Law equilibria. The former materialized when the minority was sufficiently small. By contrast, when the minority was large, all groups eventually converged to a Duverger's Law equilibrium. Under AV, and as predicted, some voters double voted to increase the vote shares of both majority candidates but they kept aggregating information. We observed extremely strong welfare performance of AV in the lab: the subjects' behavior allowed them to elect the full information Condorcet winner with a probability very close to what a social planner would have achieved after observing all available signals. However, to achieve this, subjects did not behave exactly as predicted by theory: some voters specialized in double or single voting, and the amount of double voting was lower than predicted. This led us to analyze the existence of asymmetric equilibria, which we found to have superior theoretical performance, and to better organize the data than symmetric equilibria. This might point to the need of further studying asymmetric equilibria in the information aggregation literature.

The experimental results also offer systematic support for strategic voting behavior that is consistent with the model's prediction and inconsistent with an assumption of sincere or naïve voting. Indeed, with both voting mechanisms, subjects learned how to aggregate information: they adopted an "insincere" strategy when we biased signals across states of nature. Secondly, we observed that, in line with theory, the subjects' behavior adjusted quite differently in plurality than in approval voting, leading to substantially higher realized payoffs in AV treatments.

We believe that this paper opens up many interesting theoretical and experimental 
questions about multicandidate elections. Investigating how each voting rule performs in such a setup, how plurality and approval voting perform in a setup where majority voters have mix of private and common values, or investigate deeper what induces individual voters to adapt their strategy and collectively select one or another equilibrium are just some of the potential lines of research to pursue.

\section{References}

[1] Ahn, David and Santiago Oliveros (2011a): Approval Voting and Scoring Rules with Common Values, mimeo.

[2] Ahn, David and Santiago Oliveros (2011b), The Condorcet Jur(ies) Theorem, mimeo.

[3] Ahn, David and Santiago Oliveros (2012), Combinatorial Voting, Econometrica, 80(1): 89-141.

[4] Ali, N., Goeree, J., Kartik, N. and T. Palfrey (2008), Information Aggregation in Ad Hoc and Standing Committees, American Economic Review, Papers \& Proceedings, 98(2): 181-186.

[5] Austen-Smith, David and Jeffrey S. Banks (1996), Information Aggregation, Rationality, and the Condorcet Jury Theorem, American Political Science Review, 90: $34-45$.

[6] Austen-Smith, D. and T. Feddersen (2006). Deliberation, Preference Uncertainty and Voting Rules, American Political Science Review, 100(2): 209-217.

[7] Bassi, Anna (2008), "Voting Systems and Strategic Manipulation: an Experimental Study", mimeo.

[8] Battaglini, M., Morton, R. and T. Palfrey (2008), Information Aggregation and Strategic Abstention in Large Laboratory Elections. American Economic Review, Papers \& Proceedings, 98(2): 194-200.

[9] Battaglini, M., Morton, R. and T. Palfrey (2010), The Swing Voter's Curse in the Laboratory, Review of Economic Studies, 77: 61-89.

[10] Bhattacharya, S. (2012), Preference Monotonicity and Information Aggregation in Elections, mimeo.

[11] Bhattacharya, S., J. Duffy and S. T. Kim (2012). Compulsory versus Voluntary Voting: An Experimental Study. Working Paper. 
[12] Bouton, L. (2012), A Theory of Strategic Voting in Runoff Elections, American Economic Review. forthcoming.

[13] Bouton, L., and M. Castanheira (2009), The Condorcet-Duverger Trade-Off: Swing Voters and Voting Equilibria, in Aragonés, E., C.. Beviá, H. Llavador and N. Schofield, eds.: The Political Economy of Democracy. Fundacion BBVA, 121-142.

[14] Bouton, Laurent, and Micael Castanheira (2012). One Person, Many Votes: Divided Majority and Information Aggregation. Econometrica, 80(1): 43-87.

[15] Bouton, L. and G. Gratton (2012), Majority Runoff Elections: Strategic Voting and Duverger's Hypothesis, IED Discussion Paper, 241.

[16] Bouton, Laurent, Aniol Llorente-Saguer and Fréderic Malherbe (2012), Not Convicting the Innocent: Majority Rule with Veto Power, mimeo.

[17] Callander, S. (2008), Majority Rule When Voters Like to Win, Games and Economic Behavior, 64: 393-420.

[18] Castanheira, M. (2003), Why Vote for Losers?, Journal of the European Economic Association, 1: 1207-1238.

[19] Clotfelder, C. and P. Cook (1993), The "Gambler's Fallacy" in Lottery Play, Management Science, 39:1521-1525.

[20] Croson, R. and Sundali, J. (2005), The Gambler's Fallacy and the Hot Hand: Empirical Data from Casinos, Journal of Risk and Uncertainty, 30: 195-209.

[21] de Condorcet, N.C. (1785). Essai sur l'Application de l'Analyse à la Probabilité des Decisions Rendues à la Pluralité des Voix. Paris.

[22] Coughlan, P. (2001). In Defense of Unanimous Jury Verdicts: Mistrials, Communication and Strategic Voting, American Political Science Review, 94: 375-93.

[23] Cox, Gary (1997). Making Votes Count. Cambridge, UK: Cambridge University Press.

[24] Duverger, M. (1963), Political Parties: their Organization and Activity in the Modern State, North, B. and North R., tr. New York: Wiley, Science Ed.

[25] Ellis, A. (2012), Condorcet Meets Ellsberg, mimeo.

[26] Eraslan, H. and P. Bond (2009), Strategic Voting over Strategic Proposals, Review of Economic Studies, 77(2): 459-490.

[27] Feddersen, T., Gailmard, S. and A. Sandroni. (2009), Moral Bias in Large Elections: Theory and Experimental Evidence, American Political Science Review, 103(2):175192. 
[28] Feddersen, T. and Pesendorfer, W. (1996), The Swing Voter's Curse, American Economic Review, 86: 408-424.

[29] Feddersen, T. and Pesendorfer, W. (1997), Voting Behavior and Information Aggregation in Elections with Private Information, Econometrica, 65: 1029-1058.

[30] Feddersen, T. and Pesendorfer, W. (1998), Convicting the Innocent: the Inferiority of Unanimous Jury Verdicts, American Political Science Review, 92: 23-35.

[31] Fey, Mark (1997) Stability and Coordination in Duverger's Law: A Formal Model of Pre-election Polls and Strategic Voting. American Political Science Review, 91(1): $135-47$.

[32] Fey, M. and Kim, J. (2007), The Swing Voter's Curse with Adversarial Preferences, Journal of Economic Theory, 135: 236-252.

[33] Fiorina, M. and C. Plott (1978), Committee Decisions Under Majority Rule: An Experimental Study, American Political Science Review, 72: 575-98.

[34] Fischbacher, U. (2007), "z-Tree - Zurich Toolbox for Readymade Economic Experiments", Experimental Economics, 10, 171-178.

[35] Forsythe, Robert, Roger B. Myerson, Thomas A. Rietz and Robert J. Weber (1993), "An Experiment on Coordination in Multi-Candidate Elections: The Imortance of Polls and Election Histories", Social Choice and Welfare, 10: 223-247.

[36] Forsythe, Robert, Roger B. Myerson, Thomas A. Rietz and Robert J. Weber (1996), An Experimental Study of Voting Rules and Polls in Three-Way Elections. International Journal of Game Theory, 25: 355-83.

[37] Gerardi, D. (2000), Jury Verdicts and Preference Diversity, American Political Science Review, 94(2): 395-406.

[38] Gerber, Elisabeth A., Rebecca B. Morton, and Thomas A. Rietz. (1998). Minority Representation in Multimember Districts. American Political Science Review 92: 12744.

[39] Goeree, Jacob and Leeat Yariv (2011), An Experimental Study of Collective Deliberation, Econometrica 79(3): 893-921.

[40] Goertz, J. and Maniquet, F. (2009), On the Informational Efficiency of Simple Scoring Rules, Journal of Economic Theory, 146(4): 1464-1480.

[41] Granic, D.-G. (2012), The Case of Divided MAjority: Information Aggregation and Uncertianty, mimeo. 
[42] Greiner, Ben (2004), An Online Recruitment System for Economic Experiments, in Forschung und Wissenschaftliches Rechnen 2003, ed. by K. Kremer and V. Macho. GWDG Bericht 63. Gesellschaft für Wissenschaftliche Datenverarbeitung Göttingen: Datenverarbeitung, 79-93.

[43] Guarnaschelli, S., McKelvey, R., and T. Palfrey (2000), An Experimental Study of Jury Decision Rules, American Political Science Review, 94(2): 407-423.

[44] Holt, Charles A. and Susan K. Laury (2002), "Risk Aversion and Incentive Effects", American Economic Review 92, 1644-1655.

[45] Krishna, V. and J. Morgan (2011), Overcoming Ideological Bias in Elections, Journal of Political Economy, 119(2): 183-211.

[46] Krishna, V. and J. Morgan (2012), Voluntary Voting: Costs and Benefits, Journal of Economic Theory, forthcoming.

[47] Ladha, Krishna, Gary Miller, and Joseph Oppenheimer. 1996. "Information Aggregation by Majority Rule: Theory and Experiments."Washington University. Typescript.

[48] Laslier, J.-F. (2010), The Leader Rule: A Model of Strategic Approval Voting in a Large Electorate, Journal of Theoretical Politics, 22(1): 64-84.

[49] Laslier, J.-F. (2010), Laboratory Experiments on Approval Voting, in Handbook on Approval Voting, ed. by Laslier J.-F. and Sanver R, Springer-Verlag: Berlin Heidelberg

[50] Laslier, J.-F., and Van der Straeten, K. (2008), A Live Experiment on Approval Voting, Experimental Economics, 11: 97-105.

[51] Mandler, Michael (2012). "The fragility of information aggregation in large elections". Games and Economic Behavior 74: 257-268.

[52] Martinelli, C. (2002), Simple Plurality vs. Plurality Runoff with Privately Informed Voters, Social Choice and Welfare, 19: 901-920.

[53] Martinelli, C. (2006), Would Rational Voters Acquire Costly Information?, Journal of Economic Theory, 129: 225-251.

[54] McLennan, A. (1998), Consequences of the Condorcet Jury Theorem for Beneficial Information Aggregation by Rational Agents, American Political Science Review, 92, 413-418.

[55] McMurray, J. (2012), Aggregating Information by Voting: The Wisdom of the Experts versus the Wisdom of the Masses, Review of Economic Studies, forthcoming. 
[56] Morton, R, and T. Rietz (2008), Majority Requirements and Minority Representation, New York University Annual Survey of American Law, 63: 691-726.

[57] Morton, R. and J.-R. Tyran, (2012), Ethical vs Selfish Motivations and Turnout in Small and Large Elections, mimeo.

[58] Myatt, D. (2007), On the Theory of Strategic Voting, Review of Economic Studies, 74: $255-281$.

[59] Myerson, R. (2000), "Theoretical Comparison of Electoral Systems", European Economic Review, 43: 671-697.

[60] Myerson, R. (2002), "Comparison of Scoring Rules in Poisson Voting Games", Journal of Economic Theory, 103: 219-251.

[61] Myerson, R. and R. Weber, (1993), "A Theory of Voting Equilibria," American Political Science Review, 77: 102-14.

[62] Nagel, Jack (2007). "The Burr Dilemma in Approval Voting." Journal of Politics, 69, pp. $43-58$.

[63] Niemi, R.G. (1984), "The Problem of Strategic Behavior under Approval Voting", American Political Science Review, 78(4): 952-958

[64] Nuñez, M. (2009), Condorcet Consistency of Approval Voting: A Counter Example in Large Poisson Games, Journal of Theoretical Politics, 22(1): 64-84.

[65] Oliveros, S. (2011), Aggregation of Endogenous Information in Large Elections, mimeo.

[66] Palfrey, T. (1989), A Mathematical Proof of Duverger's Law, In Models of Strategic Choice in Politics, edited by P. C. Ordeshook. Ann Arbor, Michigan: University of Michigan Press.

[67] Palfrey, Thomas R. (2012), Experiments in Political Economy, forthcoming in Handbook of Experimental Economics Vol. 2 (J. Kagel and A. Roth, eds.), Princeton University Press.

[68] Piketty, T. (1999), The Information-Aggregation Approach to Political Institutions, European Economic Review, 43 (4-6): 791-800.

[69] Piketty, T. (2000), Voting as Communicating, Review of Economic Studies, 67: 169191.

[70] Rietz, T. (2008), Three-Way Experimental Election Results: Strategic Voting, Coordinated Outcomes and Duverger's Law, Handbook of Experimental Economics. 
[71] Riker, W. (1982), The Two-Party System and Duverger's Law: An Essay on the History of Political Science, American Political Science Review, 84: 1077-1101.

[72] Saari, D. and J. Van Newenhizen (1988), "The problem of indeterminacy in approval, multiple, and truncated voting systems", Public Choice 59: 101-120

[73] Tversky, Amos and Daniel Kahneman. 1971. "Belief in the Law of Small Numbers," Psychology Bulletin, 76(2), pp. 105-10.

[74] Van der Straeten, K., Laslier, J.-F., Sauger, N., and Blais, A. (2010), Strategic, Sincere, and Heuristic Voting under Four Election Rules: An Experimental Study, Social Choice and Welfare, 35: 435-472.

\section{Appendices}

\section{Appendix A1: Pivot Probabilities and Correcting Factors in AV}

The pivotal event $\operatorname{piv}_{A C}^{A V}$ is defined as follows:

$$
\begin{aligned}
& x_{A}>x_{B}-1 \text { and } x_{A}+x_{A B} \in\left\{n_{C}-1, n_{C}\right\} \\
& x_{A}=x_{B} \text { and } x_{A}+x_{A B}=n_{C}, \text { or } \\
& x_{A}=x_{B}-1 \text { and } x_{B}+x_{A B}=n_{C} .
\end{aligned}
$$

Therefore, the probability of event piv ${ }_{A C}$ in state $\omega$ is:

$$
\begin{aligned}
\pi_{A C}^{\omega} \equiv \operatorname{Pr}\left(p^{\omega} v_{A C}^{A V} \mid \omega\right)=\quad & (n-1) ! \sum_{i=0}^{1} \sum_{x_{A B}=0}^{2\left(n_{C}-i\right)-n} \frac{\left(\tau_{A}^{\omega}\right)^{n_{C}-i-x_{A B}}\left(\tau_{A B}^{\omega}\right)^{x_{A B}}\left(\tau_{B}^{\omega}\right)^{(n-1)-\left(n_{C}-i\right)}}{2\left(n_{C}-i-x_{A B}\right) ! x_{A B} !\left(n-1-n_{C}+i\right) !} \\
& +\frac{(n-1) !}{3} \frac{\left[\tau_{A}^{\omega} \tau_{B}^{\omega}\right]^{n-1-n_{C}}\left(\tau_{A B}^{\omega}\right)^{2 n_{C}+1-n}}{\left[\left(n-1-n_{C}\right) !\right]^{2}\left(2 n_{C}-n+1\right) !} \\
& +\frac{(n-1) !}{6} \frac{\left(\tau_{A}^{\omega}\right)^{n-1-n_{C}}\left(\tau_{A B}^{\omega}\right)^{2 n_{C}-n}\left(\tau_{B}^{\omega}\right)^{n-n_{C}}}{\left(n-1-n_{C}\right) !\left(n-n_{C}\right) !\left(2 n_{C}-n\right) !} .
\end{aligned}
$$

The pivot probabilities of event $p i v_{B C}^{\omega}, p i v_{A B}^{\omega}$, and $p i v_{B A}^{\omega}$ can be defined and computed similarly. In particular:

$$
\begin{aligned}
\pi_{B C}^{\omega} \equiv \operatorname{Pr}\left(p i v_{B C}^{A V} \mid \omega\right)= & (n-1) ! \sum_{i=0}^{1} \sum_{x_{A B}=0}^{2\left(n_{C}-i\right)-n} \frac{\left(\tau_{A}^{\omega}\right)^{(n-1)-\left(n_{C}-i\right)}\left(\tau_{A B}^{\omega}\right)^{x_{A B}}\left(\tau_{B}^{\omega}\right)^{n} C^{-i-x_{A B}}}{2\left(n_{C}-i-x_{A B}\right) ! x_{A B} !\left(n-1+i-n_{C}\right) !} \\
& +\frac{(n-1) !}{3} \frac{\left[\tau_{A}^{\omega} \tau_{B}^{\omega}\right]^{n-1-n_{C}}\left(\tau_{A B}^{\omega}\right)^{2 n_{C}+1-n}}{\left[\left(n-1-n_{C}\right) !\right]^{2}\left(2 n_{C}-n+1\right) !} \\
& +\frac{(n-1) !}{6} \frac{\left(\tau_{A}^{\omega}\right)^{n-n_{C}}\left(\tau_{A B}^{\omega}\right)^{2 n_{C}-n}\left(\tau_{B}^{\omega}\right)^{n-n_{C}-1}}{\left(n-1-n_{C}\right) !\left(n-n_{C}\right) !\left(2 n_{C}-n\right) !},
\end{aligned}
$$




$$
\begin{aligned}
\pi_{A B}^{\omega} \equiv \operatorname{Pr}\left(p i v_{A B}^{\omega} \mid \omega\right)=\quad & (n-1) ! \sum_{i=0}^{1} \sum_{k=i}^{n+2(i-1)-n_{C}} \frac{\left(\tau_{A}^{\omega}\right)^{k-i}\left(\tau_{A B}^{\omega}\right)^{n+(i-1)-2 k}\left(\tau_{B}^{\omega}\right)^{k}}{2 k !(k-i) !(n+i-1-2 k) !} \\
& +\frac{(n-1) !}{3} \frac{\left[\tau_{A}^{\omega} \tau_{B}^{\omega}\right]^{n-1-n_{C}}\left(\tau_{A B}^{\omega}\right)^{2 n_{C}+1-n}}{\left[\left(n-1-n_{C}\right) !\right]^{2}\left(2 n_{C}-n+1\right) !} \\
& +\frac{(n-1) !}{6} \frac{\left(\tau_{A}^{\omega}\right)^{n-1-n_{C}}\left(\tau_{A B}^{\omega}\right)^{2 n_{C}-n}\left(\tau_{B}^{\omega}\right)^{n-n_{C}}}{\left(n-1-n_{C}\right) !\left(n-n_{C}\right) !\left(2 n_{C}-n\right) !}
\end{aligned}
$$

and

$$
\begin{aligned}
\pi_{B A}^{\omega} \equiv \operatorname{Pr}\left(p_{i v}^{A V} \mid \omega\right)=\quad & (n-1) ! \sum_{i=0}^{1} \sum_{k=i}^{n+2(i-1)-n_{C}} \frac{\left(\tau_{A}^{\omega}\right)^{k}\left(\tau_{A B}^{\omega}\right)^{n+(i-1)-2 k}\left(\tau_{B}^{\omega}\right)^{k-i}}{2 k !(k-i) !(n+i-1-2 k) !} \\
& +\frac{(n-1) !}{3} \frac{\left[\tau_{A}^{\omega} \tau_{B}^{\omega}\right]^{n-1-n_{C}}\left(\tau_{A B}^{\omega}\right)^{2 n_{C}+1-n}}{\left[\left(n-1-n_{C}\right) !\right]^{2}\left(2 n_{C}-n+1\right) !} \\
& +\frac{(n-1) !}{6} \frac{\left(\tau_{A}^{\omega}\right)^{n-n_{C}}\left(\tau_{A B}^{\omega}\right)^{2 n_{C}-n}\left(\tau_{B}^{\omega}\right)^{n-n_{C}-1}}{\left(n-1-n_{C}\right) !\left(n-n_{C}\right) !\left(2 n_{C}-n\right) !}
\end{aligned}
$$

Using the pivot probabilities and the expected gains of the different actions (i.e. (12), (13), and (14)), we can compute the correcting factors $\phi^{a}$ and $\phi^{b}:{ }^{4}$

$$
\begin{aligned}
\phi^{a}= & {\left[\operatorname{Pr}\left(X_{A}=X_{B}=n_{C}-1 \mid a\right)(V+v)+\operatorname{Pr}\left(X_{A}=X_{B}+1=n_{C} \mid a\right) v+\ldots\right.} \\
& \left.\ldots+\operatorname{Pr}\left(X_{A}+1=X_{B}=n_{C} \mid a\right) V+\operatorname{Pr}\left(X_{A}=X_{B}=n_{C} \mid a\right)(V+v)\right] / 6, \text { and } \\
\phi^{b}= & {\left[\operatorname{Pr}\left(X_{A}=X_{B}=n_{C}-1 \mid b\right)(V+v)+\operatorname{Pr}\left(X_{A}=X_{B}+1=n_{C} \mid b\right) V+\ldots\right.} \\
& \left.\ldots+\operatorname{Pr}\left(X_{A}+1=X_{B}=n_{C} \mid b\right) v+\operatorname{Pr}\left(X_{A}=X_{B}=n_{C} \mid b\right)(V+v)\right] / 6 .
\end{aligned}
$$

We directly see that $\phi^{\omega}=0$ when $\tau_{A B}^{\omega} \in\{0,1\}$, or $\tau_{A}^{\omega}=0$, or $\tau_{B}^{\omega}=0$.

\subsection{Appendix A2: Plurality, Equilibrium Analysis}

Proof of Proposition 3. Consider a distribution of signals such that $r\left(s_{A} \mid a\right)-r\left(s_{B} \mid b\right)>$ $\delta\left(n, n_{c}\right)$, in which case sincere voting is not an equilibrium. That is, there exists a signal $\bar{s} \in$ $\left\{s_{A}, s_{B}\right\}$ such that all the voters who received signal $\bar{s}$ strictly prefer to deviate from a strategy profile $\sigma^{\text {sincere }} \equiv\left\{\sigma_{s_{A}}(A), \sigma_{s_{B}}(B)\right\}=\{1,1\}$.

Case 1: $\bar{s}=s_{A}$. In this case, $\sigma^{\text {sincere }} \Rightarrow G\left(A \mid s_{A}\right)-G\left(B \mid s_{A}\right)<0$. Now, consider a second strategy profile $\sigma^{\prime} \equiv\left\{\left[r\left(s_{A} \mid a\right)+r\left(s_{A} \mid b\right)\right]^{-1}, 1\right\}$. With this profile, we have: $\tau_{A}^{a}=\tau_{B}^{b}$ and $\tau_{A}^{b}=\tau_{B}^{a}$, and thus $p_{B C}^{b}=p_{A C}^{a}>0$ and $p_{A C}^{b}=p_{B C}^{a}>0$ and, from (9):

$$
G(A \mid s)-G(B \mid s)=\left[V p_{A C}^{a}-v p_{B C}^{a}\right][q(a \mid s)-q(b \mid s)]
$$

where (i) $\left[V p_{A C}^{a}-v p_{B C}^{a}\right]$ is positive, and (ii) $[q(a \mid s)-q(b \mid s)]$ is positive for $s_{A}$ and negative for $s_{B}$. In other words, all voters would strictly prefer to deviate from $\sigma^{\prime}$ by voting sincerely.

\footnotetext{
${ }^{44}$ Proof available upon request.
} 
This means that the value of $G\left(A \mid s_{A}\right)-G\left(B \mid s_{A}\right)$ changes sign when $\sigma_{s_{A}}(A)$ is increased from $\left[r\left(s_{A} \mid a\right)+r\left(s_{A} \mid b\right)\right]^{-1}$ to 1.

Since all pivot probabilities are continuous in $\sigma_{s_{A}}$, the differential $G\left(A \mid s_{A}\right)-G\left(B \mid s_{A}\right)$ is also

continuous in $\sigma_{s_{A}}$. This implies that there must exist a value $\sigma_{s_{A}}^{*}(A) \in\left(\left[r\left(s_{A} \mid a\right)+r\left(s_{A} \mid b\right)\right]^{-1}, 1\right)$ such that voters with signal $s_{A}$ are indifferent between playing $A$ and $B$.

Now, we prove that the strategy profile $\left\{\sigma_{s_{A}}(A), \sigma_{s_{B}}(B)\right\}=\left\{\sigma_{s_{A}}^{*}(A), 1\right\}$ is an equilibrium. This profile implies: $\tau_{A}^{a} \in\left(\frac{r\left(s_{A} \mid a\right)}{r\left(s_{A} \mid a\right)+r\left(s_{A} \mid b\right)}, r\left(s_{A} \mid a\right)\right)$ and $\tau_{B}^{b} \in\left(r\left(s_{B} \mid b\right), \frac{r\left(s_{A} \mid a\right)}{r\left(s_{A} \mid a\right)+r\left(s_{A} \mid b\right)}\right)$ and hence:

$$
\tau_{A}^{a}>\tau_{B}^{b}>\frac{n_{C}}{n}>\tau_{A}^{b}>\tau_{B}^{a} ; p_{A C}^{a}>p_{B C}^{a} \text { and } p_{B C}^{b}>p_{A C}^{b} .
$$

Since $G\left(A \mid s_{A}\right)-G\left(B \mid s_{A}\right)=0$ for that strategy profile, we know from (9) that:

$$
q\left(a \mid s_{A}\right)\left[V p_{A C}^{a}-v p_{B C}^{a}\right]=q\left(b \mid s_{A}\right)\left[V p_{B C}^{b}-v p_{A C}^{b}\right]
$$

where both sides of the equality are strictly positive. Since $q\left(a \mid s_{B}\right)<q\left(a \mid s_{A}\right),(19)$ implies:

$$
q\left(a \mid s_{B}\right)\left[V p_{A C}^{a}-v p_{B C}^{a}\right]<q\left(b \mid s_{B}\right)\left[V p_{B C}^{b}-v p_{A C}^{b}\right]
$$

which means that a voter who received signal $s_{B}$ strictly prefers to play $B$.

Case 2: $\bar{s}=s_{B}$. In this case, $\sigma^{\text {sincere }} \Rightarrow G(A \mid s)-G(B \mid s)>0$ for both signals. Now, consider another strategy profile $\sigma^{\prime \prime} \equiv\{\varepsilon, 1\}$, with $\varepsilon \rightarrow 0$ (and hence $\sigma_{s_{A}}(B) \rightarrow 1$ ). From Proposition 1 , this strategy profile implies $G(A \mid s)-G(B \mid s)<0$ for both signals. By the continuity of the payoffs with respect to $\sigma_{s_{A}}(A)$, there must therefore exist a value $\sigma_{s_{A}}^{* *}(A) \in(0,1)$ such that $G\left(A \mid s_{A}\right)-G\left(B \mid s_{A}\right)=0$ and, by the same argument as in $(19-20), G\left(A \mid s_{B}\right)-G\left(B \mid s_{B}\right)<0$. Hence, the strategy profile $\left\{\sigma_{s_{A}}(A), \sigma_{s_{B}}(B)\right\}=\left\{\sigma_{s_{A}}^{* *}(A), 1\right\}$ is an equilibrium.

Note that sincere stability is not a binding restriction, since all voters vote for their preferred alternative with a probability strictly larger than 0 .

\section{Appendix A3: Approval Voting, Equilibrium Analysis}

Lemma 2 If there exists a signal s such that

$$
\begin{aligned}
& G^{A V}(A \mid s)-G^{A V}(A B \mid s)=0 \text { then } G^{A V}\left(A \mid s_{A}\right)-G^{A V}\left(A B \mid s_{A}\right)>G^{A V}\left(A \mid s_{B}\right)-G^{A V}\left(A B \mid s_{B}\right) \\
& G^{A V}(B \mid s)-G^{A V}(A B \mid s)=0 \text { then } G^{A V}\left(B \mid s_{B}\right)-G^{A V}\left(A B \mid s_{B}\right)>G^{A V}\left(B \mid s_{A}\right)-G^{A V}\left(A B \mid s_{A}\right), \text { and } \\
& G^{A V}(A \mid s)-G^{A V}(B \mid s)=0 \text { then } G^{A V}\left(A \mid s_{A}\right)-G^{A V}\left(B \mid s_{A}\right)>G^{A V}\left(A \mid s_{B}\right)-G^{A V}\left(B \mid s_{B}\right)
\end{aligned}
$$

Proof. We detail the proof for (21). It is similar for the other two implications. Remember that 
the second term in (15) is necessarily negative. Thus $G^{A V}(A \mid s)-G^{A V}(A B \mid s)=0$ implies that the first term must be strictly positive. It follows immediately that:

$$
G^{A V}(A \mid s)-G^{A V}(A B \mid s) \geq 0 \text { iff } \frac{q(a \mid s)}{q(b \mid s)} \geq \frac{\pi_{A B}^{b}(V-v)+\pi_{B C}^{b} V-\phi^{b}}{\pi_{A B}^{a}(V-v)-\pi_{B C}^{a} v+\phi^{a}} .
$$

Thus, (21) follows from $\frac{q\left(a \mid s_{A}\right)}{q\left(b \mid s_{A}\right)}>\frac{q\left(a \mid s_{B}\right)}{q\left(b \mid s_{B}\right)}$.

Lemma 3 In any voting equilibrium under $A V$, neither $A$ nor $B$ can be approved by all voters.

Proof. We prove the proposition by contradiction and for the limit case in which $\varepsilon=0$. By definition the results hold when $\varepsilon>0$.

Policy $A$ is approved by all voters if and only if $\sigma_{s_{A}}(A)+\sigma_{s_{A}}(A B)=1=\sigma_{s_{B}}(A)+\sigma_{s_{B}}(A B)$. In this case, we have: $x_{A}+x_{A B}=n$ and hence $\pi_{A C}^{\omega}=0=\pi_{B C}^{\omega}$ and $\phi^{\omega}=0$. The only possible pivot events are when $x_{A B}=n-1$ or $n-2$. Hence:

$$
\begin{aligned}
G(A \mid s)-G(A B \mid s) & =\left[q(a \mid s) \pi_{A B}^{a}-q(b \mid s) \pi_{A B}^{b}\right](V-v) \gtrless 0 \\
G(B \mid s)-G(A B \mid s) & =\left[q(b \mid s) \pi_{B A}^{b}-q(a \mid s) \pi_{B A}^{a}\right](V-v) \gtrless 0 .
\end{aligned}
$$

with:

$$
\pi_{A B}^{\omega}=\frac{\left(\tau_{A B}^{\omega}\right)^{n-1}}{2}, \text { and } \pi_{B A}^{\omega}=\frac{\left(\tau_{A B}^{\omega}\right)^{n-2}}{2}\left[(n-1)+(2-n) \tau_{A B}^{\omega}\right]
$$

Therefore,

$$
\begin{aligned}
\frac{\pi_{B A}^{b}}{\pi_{B A}^{a}} & =\left(\frac{\tau_{A B}^{b}}{\tau_{A B}^{a}}\right)^{n-2} \frac{(n-1)+(2-n) \tau_{A B}^{b}}{(n-1)+(2-n) \tau_{A B}^{a}}, \\
\frac{\pi_{A B}^{a}}{\pi_{A B}^{b}} & =\left(\frac{\tau_{A B}^{a}}{\tau_{A B}^{b}}\right)^{n-1}
\end{aligned}
$$

Now, we show that $\frac{\pi_{B A}^{b}}{\pi_{B A}^{a}}$ is increasing in $\frac{\tau_{A B}^{b}}{\tau_{A B}^{a}}$ (from (25), it is straightforward that $\frac{\pi_{A B}^{a}}{\pi_{A B}^{b}}$ is also increasing in $\left.\frac{\tau_{A B}^{a}}{\tau_{A B}^{b}}\right)$. Taking logs, we have that the right-hand side of (24) is

$$
(n-2)\left[\log \tau_{A B}^{b}-\log \tau_{A B}^{a}\right]+\log \left[(n-1)+(2-n) \tau_{A B}^{b}\right]-\log \left[(n-1)+(2-n) \tau_{A B}^{a}\right]
$$

Differentiating with respect to $\tau_{A B}^{b}$ yields:

$$
\frac{n-2}{\tau_{A B}^{b}}-\frac{n-2}{(n-1)+(2-n) \tau_{A B}^{b}}
$$

This is non-negative if and only if $\tau_{A B}^{b} \leq 1$. Therefore, we have that $\pi_{A B}^{b}>\pi_{A B}^{a}$ and $\pi_{B A}^{b}>\pi_{B A}^{a}$ when $\tau_{A B}^{b}>\tau_{A B}^{a}$, and conversely.

We now use this result to prove that $A$ cannot be approved by all voters. From Theorem 1, Lemma 2, and Lemma 2 (in this Appendix), there are 2 cases to check: (i) $\sigma_{s_{A}}(A)=1$ and 
$\sigma_{s_{B}}(A) \in[0,1)$, and (ii) $\sigma_{s_{B}}(A)=0$ and $\sigma_{s_{A}}(A) \in(0,1]$. If $\sigma_{s_{A}}(A)=1$ and $\sigma_{s_{B}}(A) \in[0,1)$, then $\tau_{A B}^{b}>\tau_{A B}^{a}$. Hence, we have that $\pi_{B A}^{b}>\pi_{B A}^{a}$, which implies $G\left(B \mid s_{B}\right)-G\left(A B \mid s_{B}\right)>0$. Thus, there cannot be any equilibrium in which $\sigma_{s_{A}}(A)=1$ and $\sigma_{s_{B}}(A) \in[0,1)$. If $\sigma_{s_{A}}(A) \in(0,1]$ and $\sigma_{s_{B}}(A)=0$, then either $\tau_{A B}^{a}>\tau_{A B}^{b}$ or $\tau_{A B}^{a}<\tau_{A B}^{b}$. If $\tau_{A B}^{a}>\tau_{A B}^{b}$, then $\pi_{A B}^{a}>\pi_{A B}^{b}$, and thus $G\left(A \mid s_{A}\right)-G\left(A B \mid s_{A}\right)>0$. If $\tau_{A B}^{a}<\tau_{A B}^{b}$, then $\pi_{B A}^{b}>\pi_{B A}^{a}$, and thus $G\left(B \mid s_{B}\right)-G\left(A B \mid s_{B}\right)>0$. Therefore, there cannot be any equilibrium in which $\sigma_{s_{B}}(A)=0$ and $\sigma_{s_{A}}(A) \in(0,1]$.

Proof of Theorem 1. From McLennan (1998), a strategy that maximizes expected utility must be an equilibrium of such a common value game (and any finite Bayesian game like ours must have an equilibrium). Now, conjecture some strategy profile $\sigma$ that can be played under plurality. That is, $\sigma_{s}(A B)=0$ for $s=s_{A}, s_{B}$. In this case, $\pi_{A B}^{\omega}=\pi_{B A}^{\omega}=\phi^{\omega}=0<\pi_{A C}^{\omega}, \pi_{B C}^{\omega}, \omega=a, b$. Therefore, $G^{A V}(A \mid s)-G^{A V}(A B \mid s)<0$ and $G^{A V}(B \mid s)-G^{A V}(A B \mid s)<0, \forall s$. This means that $\tau_{A B}^{\omega}=0$ cannot be part of an equilibrium under $\mathrm{AV}$, and that the welfare-maximizing equilibrium under AV must produce strictly higher expected utility than plurality.

It remains to show that this equilibrium is sincerely stable. We actually show the stronger statement that, to maximize expected welfare, a strategy must satisfy $\sigma_{s_{A}}(A), \sigma_{s_{B}}(B)>0$. We show this by contradiction: suppose that $\hat{\sigma}$ maximizes expected welfare and is such that $\hat{\sigma}_{s_{A}}(A)=$ 0 . By Lemma 3, we have $\tau_{A}^{\omega}, \tau_{B}^{\omega}, \tau_{A B}^{\omega}>0$ and hence $\hat{\sigma}_{s_{B}}(A)>0$. Then, compare $\hat{\sigma}$ with some other strategy $\sigma^{\prime}$ in which $s_{A}$-voters transfer some of their votes from $B$ towards $A B$, whereas $s_{B}$-voters adapt their voting strategy so as to maintain all vote shares unchanged in state $b^{45}$

As a result, the total vote share of $A$ in state $a$ must increase (i.e. $\tau_{A}^{a}\left(\sigma^{\prime}\right)+\tau_{A B}^{a}\left(\sigma^{\prime}\right)>$ $\left.\tau_{A}^{a}(\hat{\sigma})+\tau_{A B}^{a}(\hat{\sigma})\right)$, whereas the expected fraction of double votes increases (the total vote share of $B$ remains unchanged). As a result, in state $a$, the probability that $A$ wins must increase, whereas the probability that $C$ wins decreases weakly. In state $b$, winning probabilities are unchanged. Hence, $\hat{\sigma}$ cannot maximize expected welfare: a contradiction.

Proof of Theorem 2. We prove the Theorem in two steps. First, we show that there is no interior equilibrium in which a voter strictly mixes across the three actions $A, B$, and $A B$. Second, we show that $s_{A}$-voters never play $B$, nor $s_{B}$-voters play $A$ in an interior equilibrium. It follows that the only possible interior equilibrium is such that voters with signal $s_{A}$ mix between $A$ and $A B$, and voters with signal $s_{B}$ mix between $B$ and $A B$.

First, conjecture an equilibrium in which $\sigma_{s_{A}}(A), \sigma_{s_{A}}(B), \sigma_{s_{A}}(A B)>0$. This requires:

$$
G\left(A \mid s_{A}\right)=G\left(B \mid s_{A}\right)=G\left(A B \mid s_{A}\right) .
$$

In this case, by Lemma 2 (in this Appendix), $s_{B}$-voters must be playing $B$ with probability 1, i.e. $\sigma_{s_{B}}(B)=1$. The equilibrium is therefore not interior, a contradiction. Similarly, $s_{A}$-voters must play $A$ with probability 1 if $s_{B}$-voters strictly mix between $A, B$, and $A B$.

\footnotetext{
${ }^{45}$ If $\hat{\sigma}_{s_{A}}(A B)=1$, then one must consider a transfer of $s_{A}$-votes from $A B$ towards $A$, and $s_{B}$-voters adapt their strategy to maintain all $\tau_{\psi}^{a}$ unchanged.
} 
Second, imagine that $s_{B}$-voters play $A$ with strictly positive probability in equilibrium: $\sigma_{s_{B}}(A) \in$ $(0,1)$. This requires either (i) $G\left(A \mid s_{B}\right)=G\left(A B \mid s_{B}\right) \geq G\left(B \mid s_{B}\right)$ or (ii) $G\left(A \mid s_{B}\right)=G\left(B \mid s_{B}\right) \geq$ $G\left(A B \mid s_{B}\right)$. By Lemma 2, both (i) and (ii) imply that $G\left(A \mid s_{A}\right)>G\left(A B \mid s_{A}\right), G\left(B \mid s_{A}\right)$, and hence that $A$ 's strategy cannot be interior. By symmetry, $\sigma_{s_{A}}(B) \in(0,1)$ cannot be part of an interior equilibrium either.

\section{Appendix A4: Sample Instructions (of the main part)}

This part consists of 100 rounds. The rules are the same for all rounds. At the beginning of this part, you will be randomly assigned to a group of 12 (including yourself). You will belong to the same group throughout the whole experiment. The earnings in this part will depend partly on your decisions, partly on the decisions of the other participants in your group and partly on chance.

The Jar. There are two jars: the red jar and the blue jar. The red jar contains 6 red balls and 3 blue balls. The blue jar contains 6 blue balls and 3 red balls. At the beginning of each round, one of the two jars will be randomly selected. Each jar is equally likely to be selected, i.e., each jar is selected with a chance of $50 \%$. You will not be told which jar has been chosen when making your decision..

The Ball. After a jar is selected for your group, the computer will show each of the participants in your group (including yourself) the color of one ball randomly drawn from that jar. Since you are 12 in your group, the computer performs this random draw 12 times. Each ball will be equally likely to be drawn for every member of the group. That is, if the color of the selected jar for your group were red, then all members of your group would draw their sample balls from a jar containing 6 red and 3 blue balls. If the color of your group's jar were blue, then all members of your group would draw their sample balls from a jar containing 3 red and 6 blue balls. Therefore, if the selected jar is blue, each member of your group has a chance of 2 thirds of receiving a blue ball. And if the selected jar is red, each member of your group has a chance of 2 thirds of receiving a red ball.

But you will only see the color of your own ball. This will be the only information you will have to help you guess which jar was selected by the computer.

Your decision. Once you have seen the color of one of the balls, you can make your decision.

[Treatment Plurality] You will have to vote for Blue, Red or Black.

[Treatment Approval Voting] You will have to vote for Blue, Red, Black or any combination of them. That is, you can vote Blue, Red, Black, Blue and Red, Blue and Black, Red and Black, or Blue, Red and Black.

You can vote for one of the colors by clicking below the color. You can unvote by clicking again below the color. After making your decision, please press the 'OK' key.

Group Decision. When all participants have taken their decision, the votes of all participants will be added up. On top of that, the computer will add 7 votes for Black. The group decision will depend on the final amount of votes that each color receives:

- If one color has strictly more votes than other colors, this color will be the group decision.

- If there is a tie between several colors with the most votes, one of the colors with the most votes will be selected randomly. Each color will have the same probability of being chosen. The chosen color will be the group decision. 
Payoff in Each Round. Your payoff depends on the group decision and the color of the selected jar. Your payoff is indicated in the following table:

\begin{tabular}{c|c|c|c|c|}
\multicolumn{2}{c|}{} & \multicolumn{3}{c}{ Group Decision } \\
\cline { 3 - 5 } \multicolumn{1}{c|}{} & Blue & Red & Black \\
\cline { 2 - 5 } Jar Selected by & Blue & 200 & 110 & 20 \\
\cline { 2 - 5 } the Computer & Red & 110 & 200 & 20 \\
\cline { 2 - 5 } & &
\end{tabular}

The left part of the table indicates the group decision and the top part of the table indicates the color of the selected jar.

- If the selected jar is Blue and the group decision is Blue, you get 200 cents.

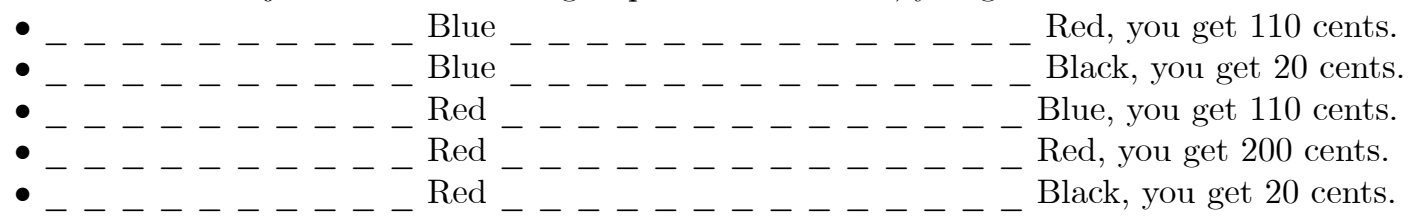

To summarize, if the color of the group decision matches the color of the selected jar, your payoff is 200. If the color of the group decision is either blue or red, but does not coincide with the color of the selected jar, your payoff is 110. Finally, if Black is the color of the group decision, your payoff is 20 .

Information at the end of each Round. Once you and all the other participants have made your choices, the round will be over. At the end of each round, you will receive the following information about the round:

- Total amount of votes for Blue

- Total amount of votes for Red

- Total amount of votes for Black (including the $7 / 9$ added by the computer)

- Group decision

- Color of the selected jar

- Your payoff

Final Earnings of this Part. At the end of this part, the computer will randomly select four rounds and you will earn the payoffs you obtained in these rounds. Each of the 100 rounds has the same chance of being selected.

Control Questions. Before starting this part, you will have to answer some control questions in the computer terminal. Click Ok after you have answered all the questions of a page. Once you and all the other participants have answered all the questions, Part 1 will start. 